\title{
Beiträge zur Tonometrie und Manometrie des Auges.
}

Ton

Dr. W. Koster Gzn.

aus Utrecht.

Experimentelle Arbeit aus dem Laboratorium von Prof. Th. Leber in Heidelberg.

Mit 9 Figuren im Text.

I.

Ueber das Fick'sche Ophthalmotonometer, nebst Angabe einer verbesserten Modification desselben.

Bei meinen Versuchen über die Folgen der Unterbindung der Venae vorticosae bei Kaninchen ${ }^{1}$ ) habe ich beinahe regelmässig das Fick'sche Tonometer (1) angewandt, um das Verhalten des Augendruckes in den verschiedenen Stadien zu prüfen. Obwohl dieses Instrument im Vergleich mit den früher construirten Tonometern einen erheblicheu Fortschritt darstellt, giebt es doch zu verschiedenen Einwänden Anlass, und es ist deshalb ein Bedürfniss, die Genauigkeit der damit vorgenommenen Messungen kennen $z u$ lernen. Ich möchte daher hier eine kurze Kritik des Instrumentes und einige damit angestellte Controlversuche folgen lassen.

Bekanntlich besteht das erwähute Tonometer aus einer

1) Dieses Heft des Archivs S. 30 ff.

v. Graefe's Archiv fur Ophthalmologie. XLI. 2. 
plangeschliffenen, runden Platte von bestimmtem Durchmesser, welche so stark auf das Auge gedrückt wird, dass ihr Rand ringsum die Bulbusoberfläche eben genau berihrt. Der Druck, der von aussen her auf die Platte ausgeuibt. wird, ist dann gleich dem Gegendruck, der im Inneren des Auges auf der plangedriickten Fläche ruht, und die Dimensionen des Apparates sind so gewählt, dass bei der angegebenen Einstellung $1 \mathrm{gr}$ Druck genau $2 \mathrm{~mm} \mathrm{Hg}$ entspricht.

1) Ein erster Fehler des Instrumentes ist, dass die Platte zu gross ist, als dass man, wie Fick annimmt, die Wandspannung der Sklera oder der Cornea vernachlässigen könnte. Ein Durchmesser der Platte von ungefähr $7 \mathrm{~mm}$ giebt bei einem Radius des Bulbus von $13 \mathrm{~mm}$ keine derartige Abplattung, die sich gewissermassen in die angrenzen-

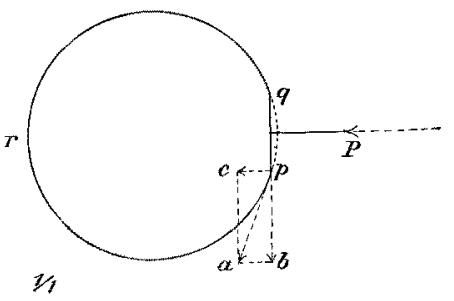

Fig. 1. den Theile der Sklera ohme merkbaren Winkel fortsetzt. In nebenstehender Zeichnung sind diese Verhältnisse in wirklicher Grösse dargestellt. In einem Punkte $p$ des Bulbus, der genau an dem Rande der Platte gelegen ist, wirkt die Wandspannung in der Richtung der Tangente $p a$, die in diesem Punkte an dem Kreise $q p r$ gezogen ist. Diese Kraft $p a$ hat einen endlichen Werth; sie ist in jedem Punkte des Umkreises der plangedrückten Flüche gleich der Kraft, womit zwei neben einander gelegene Punkte der Sklera auseinander gezogen werden. Zerlegt man die Kraft $p a$ in eine Componente $p b$, welche parallel zur Platte verläuft, und eine Componente $p c$, welche senkrecht auf derselben steht, so wird die Kraft $p b$ durch die correspondirende in dem Punkt $q$ aufgehoben, während die Kraft $p e$ dem intraocularen Druck entgegen wirkt. Die Kraft $P$, welche gefordert wird, nm die Platte eben uberall 
anzudrücken, ist also nicht gleich dem auf der Fläche $p q$ lastenden intraocularen Druck, sondern kleiner als dieser, weil die Componente $p c$ der Wandspannung ebenfalls einen Theil dieses Druckes trägt. Dieser Fehler des Instrumentes ist schwer $\mathrm{zu}$ ändern, weil eine viel kleinere Platte nur einen sehr geringen Druck in Grammen braucht, um am normalen Bulbus benutzt zu werden. Allein man könnte dann die Feder viel schwächer machen, wodurch der Ausschlag derselben wieder grösser werden würde, und sodamn Bruchtheile von Grammen genau angezeigt werden könnten; ein Gramm könnte dann z. B. 4 oder $5 \mathrm{~mm} \mathrm{Hg}$ entsprechen.

Immerhin wird aber ein Fehler bestehen bleiben, und dieser wird um so grösser sein, je kleiner der Radius der Cornea oder der Sklera ist. Besonders weil diese bei verschiedenen Individuen so sehr verschieden sind, nicht nur, wenn das Auge durch Krankheit verändert ist, sondern auch unter normalen Verhältnissen, wird dies immer ein sehr störender Factor bleiben. Einigermassen ist dem Einfluss des Unterschiedes in den Dimensionen der verschiedenen Augen dadurch abzuhelfen, dass man bei grossen Augen eine grosse und bei kleinen Augen eine kleinere Platte benutzt.

2) Ich finde bisher nicht erwähnt, dass die Dicke der Sklera oder Cornea auf das Resultat der Messung von Einfluss ist. Nehmen wir auch an, dass diese Augenhüllen absolut biegsam und unausdehnbar sind, so ist doch immer die innere Fläche derselben, auf welche die Flüssigkeit drückt, kleiner als die äussere plangedrückte Fläche. Für die Berechnung wäre also die Correction anzubringen, dass die zwischen den Radien gelegene innere Fläche mit dem Quecksilberdruck multiplicirt würde, wozu dann noch ein Theil der senkrecht auf die äussere Platte gerichteten Componente des Druckes in der Umgebung zuzuzählen wäre. Bei dem hohen specifischen Gewicht des $\mathrm{Hg}$ macht dies schon etwas aus. Ist z. B, $a b$ die Tonometerplatte, welche 
eben anliegt, so ist $a^{\prime} b^{\prime}$ die innere plangedrückte Fläche, worauf der Augendruck senkrecht lastet. Auf das angrenzende Flächenstiick $b^{\prime} c$ drückt er ebenfalls senkrecht, und zwar in jedem Punkt. Nehmen wir an, dass b'c plan ist und die Richtung der Tangente in seiner Mitte $d$ hat, so können wir den Gesammtdruck de auf die Fläche $b^{\prime} c$ zerlegen in eine Componente $d f$, die senkrecht auf die äussere Platte gerichtet ist, und in eine Componente $d y$, die parallel zu der Platte verläuft. Diese Letztere wird

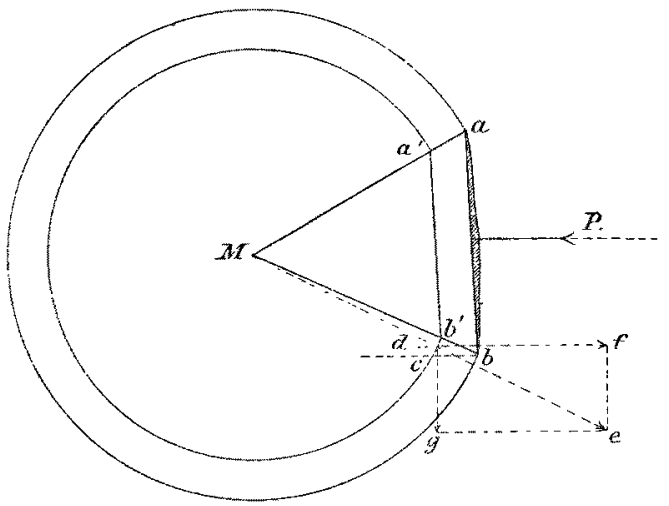

Fig. 2.

nun, in Verbindung mit derselben Componente der anderen Seite, das Bestreben haben, die innere Sklerafläche $a^{\prime} b^{\prime}$ zu dehnen und zugleich die Fläche $b^{\prime} c$ etwas der Richtung der Fläche $a^{\prime} b^{\prime}$ zu nähern, wodurch dann die Componente $d f$ auch wieder etwas vergrössert wird. Die Kraft $d f$ würde, wenn die Annahme von der Unausdehnbarkeit der Augenhüllen richtig wäre, nur von der inneren Wand des Anges getragen werden.

Für den Fall der absoluten Unausdehnbarkeit, von welchem Fick bei seinen Berechnungen ausgeht, müsste also eine Platte von der Grösse $a^{\prime} b^{\prime}$ statt $a b$ in Rechnung 
gebracht werden, woraus hervorgeht, dass durch diesen Einfluss der Augendruck ebenfalls niedriger gefunden wird, als er in Wirklichkeit ist; denn bei der Berechnung des Augendruckes wird durch Einführung eines zu grossen Werthes für die Platte, der Druck in Grammen auf jedem cbmm zu klein gefunden. Factisch sind aber die Sklera und die Cornea etwas dehnbar, und folglich wird auch ein kleiner Theil der Componente $d f$ auf die Platte $a b$ drücken.

3) Für die Begründung der Theorie geht Herr Rud. Arm. Fick von der Voraussetzung aus, dass das Auge vollkommene Kugelgestalt habe und vollkommen biegsame, aber unausdehubare Wandungen besitze. Diese Annahme ist gewiss nicht haltbar. Unter den Körpern von gleicher Oberfläche besitzt die Kugel den grössten Inhalt, folglich ist das mit Flüssigkeit gefüllt gedachte, schematische Auge mit unausdehnbarer Wandung gar nicht eindrückbar, sogar nicht, wenn der Anfangsdruck gleich Null wïre. Ein nur sehr geringer Grad von Dehnbarkeit der Wand würde zur Folge haben, dass ein kleiner Eindruck nur mit grossem Aufwand von Kraft hervorgerufen werden könnte u. s. w., wobei dann der Druck im Auge natiirlich sehr stark zunehmen müsste.

4) Am Ende der theoretischen Betrachtungen helt Fick hervor, dass es möglich wäre, dass unter dem EinHuss des Apparates der Druck im Auge sich änderte. Nachdem die Grösse des Segmentes, um welches der Inhalt des Auges nach Fick verringert wird, berechnet und als zu vernachlässigen betrachtet worden ist, meint Verfasser, dass sein Princip also einwurfsfrei dastehe. Ich glaube dagegen, dass hier der grösste Fehler des Apparates $\mathrm{zu}$ suchen ist. Denn wenn es auch richtig ist, dass der Tnhalt des Segmentes erst abgezogen werden muss, so hat Herr Fick vergessen, dass ein Druck auf die eine Seite einen Gegendruck nothwendig macht, welcher ebenfalls eine Verringerung des Inhaltes bewirkt. Der Druck 
ruht hier zwar auf einer grösseren Fläche, welche gewissermassen halbkugelige Gestalt hat, doch eine Verringerung des Inhaltes muss erfolgen und wird nicht viel von der durch die Platte verursachten versehieden sein.

5) Ueberdies tritt, weil die Skleralwand eben nicht absolut biegsam ist, eine Verkürzung des Durchmessers in der Richtung des Druckes ein, nicht nur um die Summe der Höhen der eingedrückten Theile, sondern sogar darüber, weil wegen der mangelnden Biegsamkeit der Druck der Platte sich auch auf die angrenzenden Theile der Bulbusoberfläche überträgt. Hierdurch wird der Inhalt des Bulbus weiter verkleinert, denn wenn die Kugelgestalt verloren geht, kann dieselbe Wand viel weniger Flüssigkeit in sich beherbergen. Es muss also entweder die Sklera sich ausdehnen, oder Flüssigkeit den Bulbus verlassen.

6) R. A. Fick stellt sich nun vor, dass die, aus dem Bulbussegmente fortgedrängte Flüssigkeit leicht irgendwo cinen Raum finden kann, ohne das der Augendruck erhöht wird. Denn er sagt, dass wohl ungefähr $15 \mathrm{cbmm}$ Blut und Lymphe aus dem Auge gedrängt werden (bei der Anlegung des Tonometers); doch wenn dies so wäre, würde dies schon beweisen, dass der Druck erhöht wird, denn sonst würde das Blut und die Lymphe sich überhaupt nicht fortdrängen lassen. Weiter meint er, dass durch den Tonometerdruck die Sklera etwas entspannt wird, und sich seitlich ausbiegen kann. Dies ist mir unverständlich; wie schon hervorgehoben wurde, hat die Kugel den grössten Tnhalt bei derselben Oberfläche, und ihre Formveränderung kann nur die Spannung der Wandung erhöhen. Doch besser, als hierïber zu theoretisiren, kann man versuchen, wie sich die Sache beim lebenden Thier wirklich verhält. Ich habe nun wiederholt beobachtet, dass, um $10 \mathrm{cbmm}$ Flüssigkeit in ein normales Kaninchenauge eimzupressen, ein viel höherer Druck als der bestehende nothwendig ist. Mit dem Leber'schen Manometer, einem sehr genauen In- 
strument, bei dem jeder Theilstrich der Röhren $1 \mathrm{cbmm}$ Fliissigkeit entspricht, kann man sich leicht iberzeugen, dass, wenn der Druck normal $25 \mathrm{~mm} \mathrm{Hg}$ ist, der Druck bis auf $40 \mathrm{~mm}$ gesteigert werden muss, um 10 Theilstriche Wasser ins Auge zu pressen. Bei verschiedenen Kaninchen findet man dies nun wohl etwas verschieden, aber jedenfalls muss der Druck erheblich erhöht werden. Es danert auch immer einige Minuten, bis diese Drucksteigerung sich wieder ausgeglichen hat. Nun ist zwar das Kaninchenauge kleiner als das menschliche, aber aus dem Versuch geht hervor, dass man $15 \mathrm{cbmm}$ Flüssigkeit keineswegs als eine $z u$ vernachlässigende Grösse betrachten darf.

Meiner Ansicht nach, nimmt auch die Ausdehnung der Sklera einigermassen an der Vergrösserung des Bulbusinhaltes Theil. Ich werde am Ende dieses Aufsatzes näher darauf eingehen. Ob die Abweichung ron der Kugelgestalt beim Auge eine derartige ist, dass eine blosse Formveränderung viel mehr Raum schaffen könnte, ohne dass vine Ausdehnung der Wandung hinzu käme, kann ich hier nicht entscheiden.

Ich will jetzt kurz die Controlversuche mittheilen, woraus besser, als aus allen theoretischen Betrachtungen hervorgeht, dass das Fick'sche Ophthalmotonometer über den wirklichen Druck im Auge keine ganz zuverlässige Auskunft giebt. Die von R. A. Fick und A. Fick gemachten Probeversuche sind, meiner Ansicht nach, mit ungeeigneten Instrumenten angestellt. Denn diese Experimentatoren benutzten einen Druckapparat, wobei grössere elastische Röhren, und auch eine, zum Theil mit Luft gefüllte Flasche verwendet wurden. Auch waren die Abmessungen des Apparates, soweit ich sehen kann, zu gross, um kleine Druckänderungen überhaupt anzuzeigen. Der Hauptfehler bei den Probeversuchen ist aber, dass das Auge während der Applicirung des Tonometers nicht von dem Manometer abgeschlossen wurde. Es geht aus den vorge- 
führten Zahlen nur hervor, dass die Annahme: es besteht auf beiden Seiten der Platte, wenn diese eben angedrückt ist, derselbe Druck, ungefähr richtig ist. Denn wiewohl der Manometerdruck nicht abgelesen wurde in dem Augenblick, als das Tonometer in situ war, so wurde bei den grossen Dimensionen der Röhre und Flasche, der Druck im Manometer durch die aus dem Auge austretende Flüssigkeit nur wenig gesteigert.

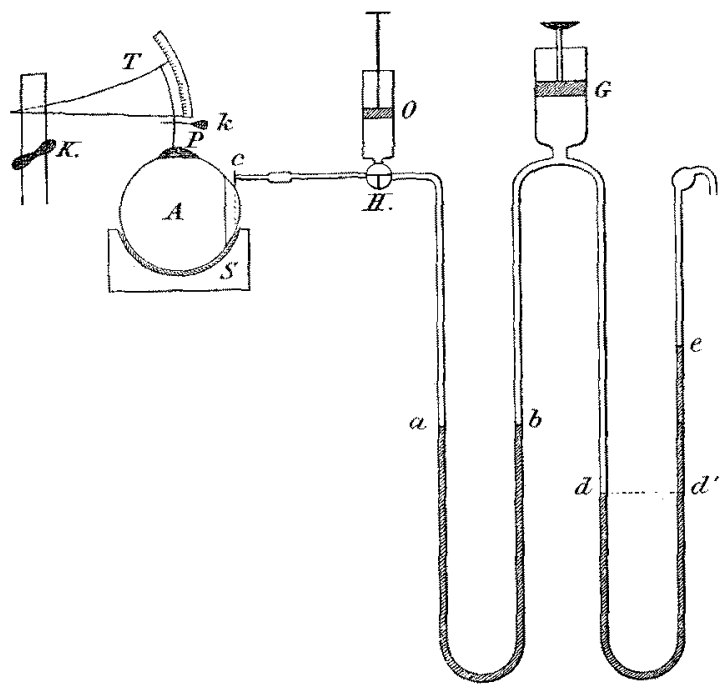

Fig. 3.

Meine Versuche sind nun auf folgende Weise angestellt worden:

Ein ganz frisches, normales Schweinsauge, liegt in einer Schale $S$, welche mit feuchtem Fliesspapier derart gefüllt ist, dass der Bulbus gleichmässig auf der ganzen unteren Fläche ruht. Die Canüle $c$ ist in die vordere Kammer gestochen worden und ist durch einen dünnen, aber dickwandigen Gummischlauch von $1 \mathrm{~cm}$ Länge mit dem Manometerrohr verbunden. Alle Luft ist entfernt, 
und ich habe mich überzeugt, dass der kleine Schlauch einen Druck von ziemlicher Höhe ohne jeglichen Fehler trägt.

In dem Auge wird jetzt mittelst der beiden Wasserreservoirs $O$ und $G$ ein Druck von $e l^{\prime} \mathrm{mm} H g$ erzeugt, und zugleich in dem ersten Rohr das Quecksilber gleich hoch, auf $a$ und $b$, gestellt. Jetzt wird der Halm $H$ so gedreht, dass das Auge abgeschlossen ist. Das Tonometer $T$, in einem Stativ $K$ aufgestellt, wird so weit vorgeschoben, bis die Platte $P$ eben anliegt, und der Druck in Grammen abgelesen. Dann wird eine Klemme $k$ angebracht, welche verhindert, dass die Platte ihren Stand wieder rerlassen kann, und jetzt schnell der Hahn geöffnet, wodurch die Communication mit dem Manometer wieder hergestellt ist. Es tritt dann Flïssigkeit aus dem Auge aus, welche $a$ und $a$ nach unten, und $b$ und $e$ nach oben drängt. Wird nun mittelst der Spitze $G$ so viel Fliissigkeit eingetrieben, bis das Quecksilber wieder auf $a$ und $b$ steht, so enthält der Bulbus wieder ebensoviel Flissigkeit als vor dem Oeffnen des Hahnes und der jetzige Druck im Manometerrohr $d d^{\prime} e$ giebt die Spannung an, welche in dem Bulbus bestand, als das Tonometer angedruickt war.

I. Ganz frisches Schweinsauge. Caniule des Manometers in der vorderen Kammer.

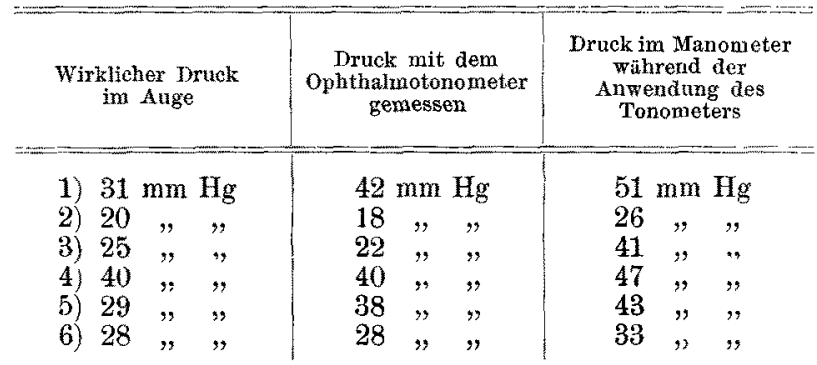

NB. Bei 1) und 2) lag das Auge auf einer planen Fläche.

II. Ganz frisches Schweinsauge. Canüle des Manometers in der vorderen Kammer. 
W. Koster.

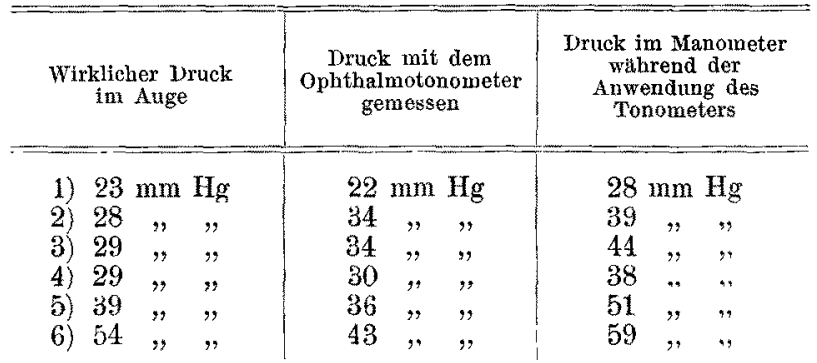

III. Ausgeräumtes Schweinsauge mit Quecksilber gefiullt. In den Opticus ist ein gebogenes Glasrohr eingebunden, um zu verhïten, dass das Queeksilber mit dem Metall des Manometers in Berührung kommt. Im Glasrohr steht das $\mathrm{Hg}$ ungefälir so hoch, wie der höchste Punkt des Bulbus. Um alle Flïssigkeit auszufiltriren, wurde der Bulbus erst 10 Minuten bei $50 \mathrm{~mm} \mathrm{Hg}$ beobachtet, dann 5 Minuten ausgerult bei $0 \mathrm{Hg}$, und dann der Versuch angefangen.

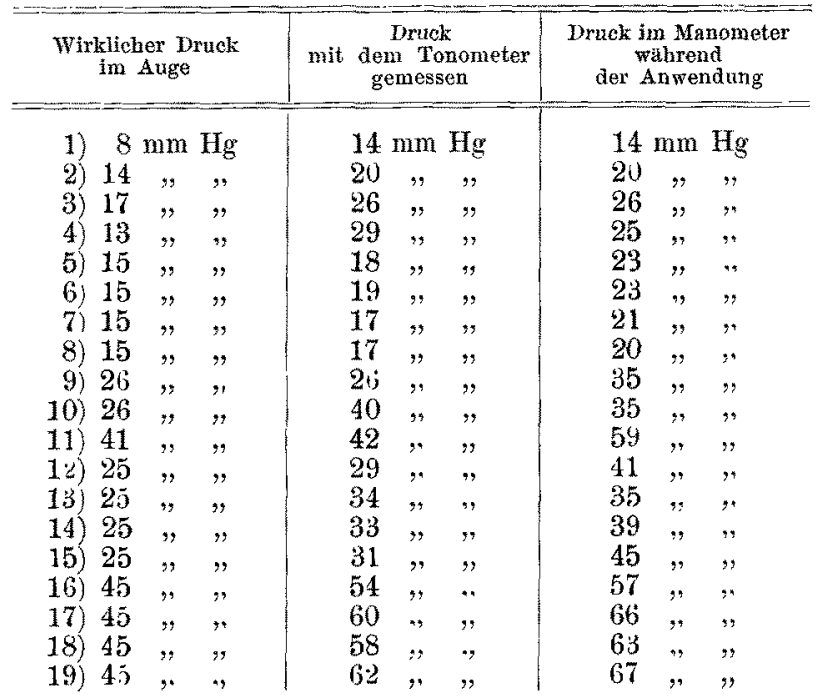

IV. Ausgeräumtes Schweinsauge mit Knochenöl gefüllt. In den Opticus ist ein nach oben stehendes Glasrohr eingebunden, so dass das Oel oben bleibt. 
Beiträge zur Tonometrie und Manometrie des Auges.

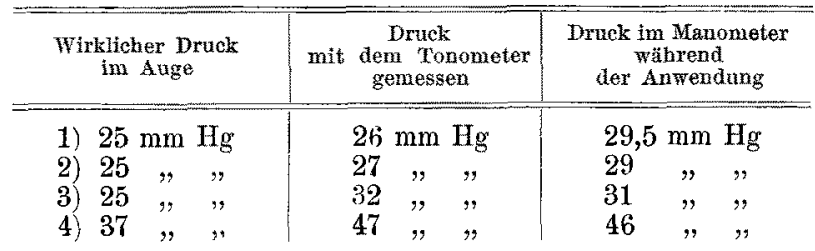

Die Versuche III und IV wurden angestellt, um einen Bulbus zu haben, der gar nicht filtrirt. Mit dem $\mathrm{Hg}$ gelang dies beinahe vollkommen. Herr Professor Leber, der mir vorschlug, den Versuch so einzurichten, meinte, dass nicht ausgeräumte Augen zu schnell filtriren würden, um genane Resultate zu geben. Die nicht ausgeräumten Augen stehen den normalen Verhältnissen aber viel näher, und desshalb haben die damit angestellten Versuche wieder mehr Werth. Bei dem mit $\mathrm{Hg}_{\mathrm{g}}$ gefüllten Auge war deutlich wahrzunehmen, dass das nicht gespannte Auge eine andere Form hatte als das gespannte. Mehr noch als bei der Betrachtung zeigte sich dies bei der Drucksteigerung mit dem Manometer, da es bei einer Druckerhöhung von $O$ auf $22 \mathrm{~nm} 89 \mathrm{cbmm}$ Quecksilber in sich aufnahm.

Betrachten wir die gewonnenen Zahlen näher, so stellt sich heraus, dass überall der Manometerdruck während der Application des Tonometers viel höher war, als im ruhenden Auge; dass also durch die Anwendung des Tonometers der Augendruck gesteigert wird. Weiter sind die Zahlen, welche das Instrument angegeben hat, ziemlich wechselnd, das eine Mal mehr mit dem wirklichen, das andere mehr mit dem geänderten Augendruck übereinstimmend, woraus mir hervorzugehen scheint, dass die Benutzung des Tonometers sehr schwierig ist, indem es nicht immer genau zu sehen ist, wann die Platte überall anliegt. Ich hatte, bevor ich diese Controlversuche anstellte, länger als 4 Monate tagtäglich mit dem Instrument gearbeitet, und die ungleichen Resultate können daher nichtzu kurzer Uebung zugeschrieben werden.

Bei den Versuchen I und II filtrirte, während der 
Beobachtung, immer etwas Flüssigkeit aus dem Auge, und es wurden also die Werthe, welche den Druck im comprimirten Auge angeben, factisch kleiner gefunden, als sie in Wirklichkeit sind. Man sieht auch, dass bei höherem Druck der Unterschied zwischen den Zahlen der ersten und der dritten Reihe kleiner ist, als bei niedrigem, was dem. schnelleren Filtriren im ersten Falle entspricht. Durch die sub. 1 und 2 genannten Einflüsse wird der intraoculare Druck zu klein gemessen, wie wir gesehen haben; durch den hier genannten Factor finden wir den Werth zu gross; die Fehler des Instrumentes heben einander also theilweise auf.

Wir bestimmen mit dem Fick'schen Ophthalmotonometer, gerade so wie mit dem tastenden Finger, ob der zu untersuchende Bulbus leicht oder schwer noch ein gewisses Quantum Flïssigkeit aufnehmen kann. Diese. mögliche Raumvergrösserung ist abhängig: 1. von der Abweichung der Form des Bulbus von der Kugelgestalt; 2. von den Elasticitätscoefficienten der Augenhüllen; 3. von dem bestehenden Druck im Auge; 4. von der Biegsamkeit der Sklera und der Cornea; 5. von der Schnelligkeit, womit etwas Flüssigkeit das Auge verlassen kann. Wenn man das Tonometer längere Zeit am Auge applicirt, so kann man die Fehler kleiner machen, indem die Druckerhöhung, welche durch die Application hervorgerufen wurde, sich allmählig ausgleicht; allein der wahre Druck kann dabei doch nicht gefunden werden. Denn, hätte man die Platte ganz angedrückt, so würde sie z. B. nach 5 Minuten in die Sklera- oder Corneafläche eingesunken sein, und hätte man sie nicht weit genug angedrückt, so würde der Druck noch zu hoch bleiben. Durch Ausprobiren während längerer Zeit könnte man dann zwar den Einfluss der meisten störenden Factoren beseitigen, allein der von der Wandspannung and der von der Dicke der Sklera und Cornea herrïhrende Fehler bleibt bestehen. Ueber den wirklichen Druck im Auge kann nur das Manometer Auf- 
schluss geben; wenn die feine Canïle richtig durch die Comea hindurchgestochen ist, sodass die Krümmmng der Hornhaut nicht geändert wird, und das Instrument ganz zuverlässig ist, so finden wir hier den wahren Druck.

Fiir gewöhmliche Versuche kann man das Manometer aber nicht anwenden, und weil wir für den, mittelst des Fingers bestimmten Druck, nur sieben Stufen haben, so scheint mir das Fick'sche Tonometer das einfachste Instrument, um die fünf oben genannten Factoren in einer Zahl auszudrücken. Wiewohl Ad. Weber eigentlich auf die Priorität der Erfindung des Primcips Anspruch machen kann, so gebuihrt doch den Herren Fick das grosse Verdienst, dasselbe in einer Weise zur Verwendung gebracht zu haben, welche die Benutzung möglichst einfach macht. Das Fick'sche Ophthalmotonometer hilft einem grossen Bedürfniss ab, welches bis jetzt, weniger in der Praxis, als wohl bei experimentellen Arbeiten gefühlt wurde.

Tch habe hier nur meine Meinung uiber das genamnte Tonometer auseinandersetzen wollen, und habe die übrigen, demselben $Z$ weck dienenden Instrumente gar nicht erwähnt. Man schliesse daraus nicht, dass ich diese als ganz werthlos betrachte. Theoretisch erreicht man mit allen ungefähr dasselbe ${ }^{1}$ ), allein das hier versuchte ist weitaus am brauchbarsten, und es wäre daher zu wünschen, dass es bei allen Experimenten über Druck und Drucksteigerung des Auges Anwendung finden möchte. Durch äusserlich auf den Bulbus applicirte Instrumente ist es, meiner Meinung nach, unmöglich, jemals den wahren Augendruck zu bestimmen.

Es ist nun neuerdings in diesem Archiv eine Arbeit erschienen von Ostwalt ${ }^{2}$, der ebenfalls das Fick'sche und auch das Maklakoff'sche Tonometer einer experimen-

D) Hierbei wird natürlich vorausgesetzt, dass die Graduirung der Scala durch Versuche an einer ahgeschlossenen Kapsel, worin der Druck bekannt ist, gewormen worden ist.

2) v. Graefe's Archiv XL. 5. (1894.) 
tellen Kritik unterzogen hat. Ostwalt hat aber, gerade wie Fick selbst, unterlassen, den Druck mittelst des Manometers in dem Augenblick zu bestimmen, wo das Tonometer in situ war. Und wiewohl er num meint, dass der durch Applicirung des Tonometers hervorgerufene Messungsfehler kaum in die Wagschale tällt, wenn die abgeplattete Stelle nicht zu gross ist, so muss ich ihm ebenfalls vorhalten, dass eine subjective Meinung über diese Frage nichts entscheiden, sondern nur das Experiment hier Aufschluss geben kann. Ich verweise daher auf das friher Mitgetheilte. Die von Ostwalt vorgenommene "geraue wissenschaftliche Controle" des Fick'schen Ophthalmotonometers kann also meiner Ansicht nach über die Brauchbarkeit dieses Instrumentes durchaus nichts entscheiden. Es geht daraus nur wieder hervor, dass das Gesetz: bei einer plangedrückten Fläche besteht auf ihren beiden Seiten derselbe Druck, annähernd richtig ist.

Die Aenderung, welche Ostwalt an dem Fick'schen Tonometer angebracht hat, nämlich der Schieber, welcher seine Stelle behält, wenn die Feder zurückgeht, scheint mir auch keine Verbesserung. Erstens ist eine viel zu grosse Kraft erforderlich, um den Schieber in Bewegung zu setzen, und zweitens geschieht es bei der Benutzung des Tonometers sehr oft, dass man etwas zu weit eindrückt und die Feder dann wieder etwas zurïckgehen lässt, ehe die richtige Stellung gefunden ist. Der Schieber würde dann aber auf dem höchsten Punkt stehen bleiben. Ein guter Indicator wäre natürlich ein grosser Vortheil, wenn man auch bei längerem Gebrauch ziemlich gut lernt, das Instrument einen Augenblick ruhig zu halten und die Scala abzulesen. Am einfachsten scheint es mir, eine kleine Vorrichtung anzubringen im Bereich des Ringfingers, wodurch die gebogene Stange, welche die Platte trägt, festgehalten werden kann, wenn der richtige Stand getroffen ist. Durch einen kleinen Hebel, der die Stange auf eine Unterlage festdriickt, ist dies leicht einzurichten. 
In der Werkstätte von Herrn Jung in Heidelberg ist nach unseren Angaben ein Tonometer angefertigt worden, welches die Fick'sche Construction der Hauptsache nach beibehält; es weicht aber erstens darin ab, dass die Feder schwächer ist, so dass die ganze Scala, welche eine Länge von $40 \mathrm{~mm}$ hat, einem Druck von $20 \mathrm{gr}$ entspricht. Die Scala ist angebracht auf der Stange, welche die Platte trägt; durch diese Anordnung wird eine Scala wie an dem Fick'schen Tonometer iiberflïssig, und die mögliche Reibung des Indicators an der Scala fällt fort. Zweitens sind dem Instrument drei Platten von verschiedener Grösse beigefügt, welche bei $1 \mathrm{gr}$ Druck resp. 2,3 und $4 \mathrm{~mm} \mathrm{Hg}$ entsprechen; die Durchmesser der Platten, welche leicht an- und abgeschraubt werden können, sind resp. 6,8, 5,6 und 4,8 mm. Auf der Scala stehen zwei Eintheilungen übereinander; bei der einen ist ein GrammTheilstrich in zwei gleiche Theile, bei der zweiten in drei gleiche Theile getheilt; dadurch wird die Ablesung wesentlich erleichtert. Drittens ist eine Fangeimrichtung an der Unterfläche angebracht, welche durch nebenstehende Zeichnung verdeutlicht wird. Die plane Stange,

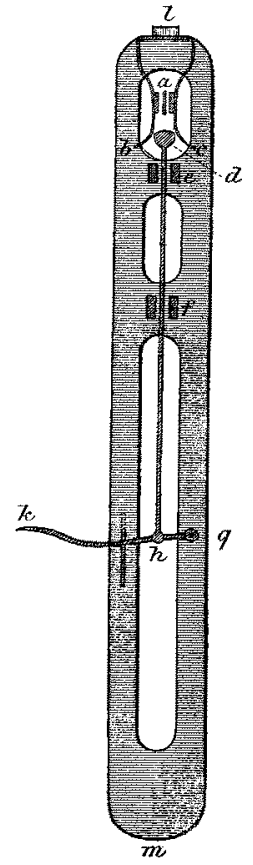

Fig. 4. worauf die Scala eingeschnitten ist und welche bei $a$ im Durchschnitt gesehen wird, bewegt sich zwischen zwei Federn $b$ und $c$, welche beim Anfang der Application des Instrumentes durch den Knopf $d$ auseinander gehalten werden. Dieser Knopf ist auf einer Stange $d h$ befestigt, welche zwischen zwei Fassungen $e$ und $f$, mittelst des Hebels $k g$ hin - und hergeschoben werden kann. Sobald die Platte richtig an dem Auge anliegt, genügt eine kleine Berührung des Hebels 
\% mit dem Ringfinger, um den Knopf $d$ zurück zu ziehen, und die Federn zusammenspringen zu lassen, wodurch die Scala $a$ in ilırem Stand fixirt wird, und der Druck nachher abgelesen werden kann. Die Länge $l m$ des Instrumentes ist etwas grösser gewählt, damit das Fnde $m$ noch in der Hohlhand gestützt werden kann. Ich glaube, dass bei dieser Form und Einrichtung des Tonometers die Anwendung besonders für Aerzte, welche nicht tagtäglich mit dem Instrument arbeiten, wesentlich erleichtert sein dürfte.

Zum Schluss dieses Abschnittes sei es mir erlaubt, noch einen Augenblick auf die Geschichte des Princips der neuen Ophthalmotonometer einzugehen. Unbestreitbar war Ad. Weber der erste, der den richtigen Weg angab. Schon im 6. Bande von Zehenders klin. Monatsbl. lesen wir S. 405: „Es kann aus der Elasticitätslehre bewiesen werden, dass bei Abflachung eines beliebigen Kugelsegmentes der elastischen Bulbushülle bis zu dessen Basis, der auf die Innenfläche dieses Segmentes wirkende Widerstand (hier der intraoculare Druck) allein getragen wird von dem die Abflachung bewirkenden Instrumente und genau ausgedrickt ist durch die Kraft, mit welcher diese Abflachung erzeugt wird. Ist es also möglich, ein Instrument herzustellen, welches bei Erzeugung einer solchen Abflachung genau den Moment anzeigt, wo dieselbe bis zur Basis des anfünglichen Kugelabschnittes gediehen ist, und zugleich die Grösse des hierzu verbrauchten Druckes registrirt, so wird damit die Aufgabe, die hydrostatischen Verhältnisse des Bulbus rein für sich zu messen, gelöst sein. Und dieser Forderung glaube ich durch mein Instrument genügt zu haben" $\left.{ }^{61}\right)$. Leider war das Letztere nicht der Fall. Ich

1) In v. Graefe's Archiv XIII. 1., p. 203 spricht Ad. Weber sich ebenfalls über ein neues Tonometer aus, jedoch ist hier von dem neeun Princip noch gar nicht die Rede. Es handelt sich hier 
kamn hier nicht näher auf die Beschreibung des Instrumentes eingehen, man sehe darïber im Handbuch von Graefe und Saemisch(5) nach, wo auch die Abbildung zu finden ist. Man wird dann auch leicht einsehen, dass bei einem weichen Bulbus z. B. die kleine mittlere Platte schon einen viel tieferen Eindruck in die Augenhüllen gemacht haben kann, als eben der Grundfläche des Stiftes entspricht, ehe die zwei Tastfüsschen auf der Sklera angelangt sind. Drückt man das Instrument so weit auf, bis die Enden der zwei Fiisschen mit der Grundfläche des Stiftes in einer Ebene liegen, so wird meistens die Sklera in der Umgebung der Tastfüsschen eingedrückt sein, und die Wandspannung ist dann nicht gleich Null. Weiter erwähnt Weber gar nicht, dass auch sein Tonometer den Druck während der Anwendung ändert; merkwürdigerweise verurtheilt er kurz vorher das Monnik'sche und später das Snellen'sche Tonometer, eben weil diese den Druck im Auge ändern. Auch Priestley Smith (13) hat dasselbe Princip aufgestellt und durch Versuche an einem Schema himreichend bestätigt gefunden; jedoch fand er, dass die Anwendung desselben in der Praxis sehr viel Schwierigkeit haben wïrle, weshall, damach kein Tonometer construint wurde.

Viel später hat Imbert(6) dieselbe Theorie, worauf das Fick'sche Tonometer beruht, auseinander gesetzt. Er führt dieselbe Formel an wie Weber; jedoch hat er sich gar nicht auf das praktische Gebiet der Tonometrie begeben. Maklakoff(2) war der Erste, der eine bessere Anwendung des Princips erfand; jedoch ist die Application seines Tonometers zeitraubend, und die damit gewonnenen Resultate sind ungenau.

Fick erfand dann beinahe zur gleichen Zeit wie Maklakoff das jetzige, einfach und leicht anzuwendende Instrument.

darum, zu beobachten, wann ein Stift anfängt, eine Formveränderung der Bulbuswand hervorzurufen.

v. Graefe's Archiv für Ophthalmologie. XII. 2. 
II.

Ein neues Tonometer.

Wiewohl die einfache Construction des Fick'schen Tonometers eine sehr zu schätzende Eigenschaft desselben ist, so bin ich nach längerer Erfahrung doch zu dem Resultat gekommen, dass bei einer etwas complicirteren Zusammenstellung ein Instrument zu machen wäre, welches auf demselben Principe berubte, und wobei sowohl die Anwendung, als die Ablesung des gefundenen Druckes noch leichter stattfinden könnte. Ich stellte an das Instrument die An= forderung, dass es selbst anzeigen muisste, wann eine Platte von einer gewissen Oberfläche eben ïberall die Sklera oder Cornea berihrt, und dass es in diesem Stande den Indicator, welcher anzeigt, wie viel Druck in Grammen dafür benuitzt worden sind, in Bezug zu der Scala fixirte, so dass man das Instrument vom Auge entfernen und dann den Druck ablesen kann.

Mittelst der folgenden Einrichtung meine ich diesen doppelten Zweck erreicht zu haben.

Eine Platte $a$ ist auf einer Stange $a b$ befestigt, diese Stange ist durch zwei Löcher in der Hülse $c d$ gefülnt und mit dieser durch die in der Zeichnung sichtbare Spiralfeder verbunden; die Letztere ist oben an der Hülse und unten an der Stange befestigt und wird also zusammengedrückt, wenn die Hülse fixirt ist und auf $"$ ein Druck lastet. Mit der Stange $a b$ ist eine dünne, platte Feder $f g h$ verbunden, welche von $n$ bis $g$ kleine Zälne trïgt und bei $h$ in einem senkrecht zu ihrer Längsrichtung gestellten Stift $h$ endet. An dem unteren Ende der Hülse ist ein Stift $e$ befestigt, der als Indicator dienen kann und welcher in dem Ruhestand der Feder $f g h$ zwischen zwei Zähne derselben eingreift. Un das untere Ende der Stange $a b$ und die Platte $a$ ist ein Cylinder angebracht; in demselben befindet sich ein Loch, durch welches der Stift $h$ durchtritt, wenn der andere Rand des Cylinders $k l$ und die Platte a gerade in einer Ebene liegen. Wird die Feder $f g h$ nach innen zurückgedrängt, so kann der Cylinder $i k l m$ uber die Platte a gesehoben werden, der Stift $h$ ruht dann gegen die innere Fläche des Cylinders und der Indicator $e$ steht frei 
von den Zähnen $n g$. Der Cylinder $i k l m$ verschiebt sich sehr leicht über die Platte $a$, und die Kraft der Feder $f g$ ist so gering, dass, wenn der Stift $h$ auf der inneren Fläche des Cylinders ruht, derselbe noch durch einen Druck von $1 \mathrm{gr}$ um die Platte verschoben werden kann. Die Reibung dex Stange $a b$ in der Hülse $a d$ ist sehr gering, auch bei horizontaler Lage des Instrumentes. Um die Hülse $g h$ ist eine längere Hülse op geschoben, welche dazu dient, das Instrumentchen wie eine Schreibfeder in der Hand festzuhalten. Einige Theile des Apparates sind aus Aluminium gearbeitet, so dass die Platte mit Stange u. s. w., welche an das Auge angedrïekt wird, noeh nicht $3 \mathrm{gr}$ wiegt. Die Seala ist für den horizontalen Stand eingesehnitten; für den verticalen Stand sind dann $3 \mathrm{gr}$ zu dem gefundenen Werth znzuzählen.

Um jetzt eine Bestimmung an dem Auge auszuführen, wird der Cylinder $i k l m$ über die Platte $a$ vorgeschoben, was einfach dadurch geschieht, dass man das Instrument vertical hält und auf die Feder $f g$ drückt. Man nimmt dann das Tonometer wie eine Schreibfeder lose in die Hand und stellt den Rand des Cylinders z. B. bei horizontaler Lage des Instrumentes auf die Cornea. Man sieht und fühlt leicht, ob der Ring überall anliegt, und dieser hat aus mechanischen Grïnden audh das Bestreben, die Cornea überall zu berülnen. Sobald dies der Fall ist, schiebt man das Tonometer in der Richtung seiner Längsaxe langsam vor; die Platte $a$ fängt dann an, den innerhalb des Ringes gelegenen Theil der Cornea allmählich plan zu drücken, und der Cylinder wird über

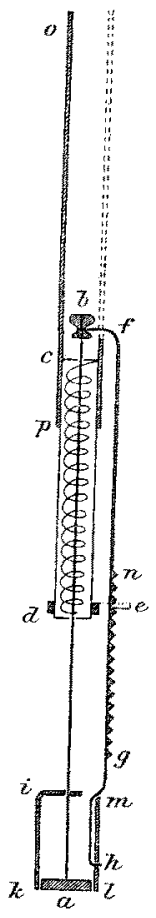

Fig. 5. die Platte zurüekgeschoben. Sobald die Platte soweit vorgedrungen ist, dass sie in einer Ebene mit dem inneren Rand des unteren Theiles des Cylinder's angekommen ist, tritt der Stift $h$ durch das Loch in dem Cylinder, die Feder $f g$ springt nach aussen und der Stift und Indicator $\theta$ wird zwischen zwei Zähnen der Feder $f g$ gefangen, wodurch die Stange sich nicht mehr gegen die Hülse verschieben kann.

Wenn man das Aussehnellen der Feder hört, nimmt man das Tonometer ab und kann auf der Scala ablesen, wie viel Druck in Grammen gebraucht worden ist. Die ganze Bestimmung dauert nur ein paar Secunden, und man kann einige hinter ein- 
ander machen, um sich von der Richtigkeit der Walırnelmung zu überzeugen.

Die Grösse der Platte ist bei diesem Instrument so gewählt, dass der Durchmesser der Oeffnung des Cylinders $6,5 \mathrm{~mm}$ misst; weil der Rand desselben natürlieh nicht absolut rechtwinkelig ist, so kann man annehmen, dass der Durchmesser des plangedrückten Theiles ungefähr $6,8 \mathrm{~mm}$ beträgt, und in dem Falle entspricht $1 \mathrm{gr}$ Druck $2 \mathrm{~mm} \mathrm{Hg}$ Druck im Auge.

\section{Prïfung des Tonometers.}

In einem Schweinsauge konnte mittelst einer dicken Glaskörpercanüle, welche nach der Einfülırung noch einige Zeit im hinteren Abschnitt des Bulbus herumgedreht wurde und welche mit einem Gummischlauch und Trichter verbunden war, jeder beliebige Druck hergestellt werden. Hart an dem Ende der Canüle lag eine Klemme, so dass, wenn dieselbe gesehlossen war, das Auge sich ganz wie eine normale abgeschlossene Augenkapsel verhielt. Der Trichter und Schlauch waren mit Kochsalzlösung von $0,75 \%$ gefült; unmittelbar vor jeder Bestimmung des Druckes mit dem Tonometer wurde die Klemme einen Augenblick geöffnet und sofort wieder geschlossen; die Höhe der Wassersäule gab also jedesmal den Druck in dem abgeschlossenen Auge an; weil die Bestimmung mit dem Tonometer auch nur ein paar Secunden in Anspruch nahm, so konnte also hier kein nennenswerther Fehler durch Filtration aus dem Auge entstehen. Ich fand folgende Werthe als Mittel von je 5 Bestimmungen.

\begin{tabular}{|c|c|c|}
\hline Druck i & im Auge & Tonometerdruck \\
\hline $5 \mathrm{~m}$ & $\mathrm{~nm} \mathrm{Hg}$ & $2,5 \mathrm{gr}$ \\
\hline & & 7,5, \\
\hline 15 & 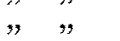 & $13 "$ \\
\hline 20 & $"$, & 19, \\
\hline 25 & $" \quad "$ & $\begin{array}{l}23,5 \\
945\end{array}$ \\
\hline
\end{tabular}

Aus dieser Tabelle geht hervor, dass bei dem Druck von $5 \mathrm{~mm} \mathrm{Hg}$ der Druck in Grammen genau die Hälfte des Quecksilberdruckes betrug. Bei den anderen Zahlen sieht man aber einen sehr starken Einfluss der Druckerhöhung im Auge durch die Anwendung des Tonometers, und zwar viel mehr hervortretend als früher bei dem Fickschen Tonometer gefunden wurde, weil hier die Bestimmung 
viel weniger Zeit erforderte, theilweise durch die andere Einrichtung des Versuches, theilweise durch die leichtere Handhabung des Tnstrumentes.

Wird das Tonometer an einem Schweinsauge versucht, das mit dem Manometer fortwährend freie Communication. behält, so werden ganz andere Werthe gefunden. In der folgenden Tabelle sind die Resultate, welche ich bei demselben Schweinsauge bekam, während zuerst das Auge mit dem Manometer in offener Verbindung stand und dann von demselben abgeschlossen wurde, neben einander eingetragen.

Statt der weiten Metalleanüle wurde hier eine noch weitere conische Glascanüle in den Glaskörperraum eingeführt; die Klemme, welche zum Abschliessen diente, lag auch hier wieder hart an dem peripheren Ende des Glasrohres.

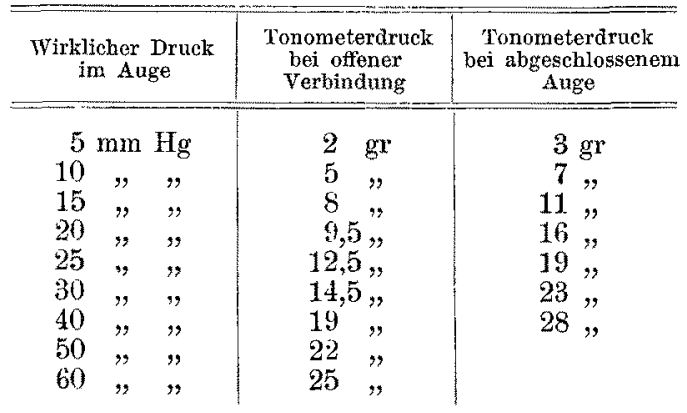

Aus diesen Zahlen ist ersichtlich, dass bis zu einem Druck von $40 \mathrm{~mm} \mathrm{Hg}$ das Tonometer den Druck im Auge, wenn dieses mit dem Manometer in offener Verbindung steht, annähernd genau angiebt, denn jedes Granm entspricht $2 \mathrm{~mm} \mathrm{Hg}$. Bei höheren Druckstufen wird der Druck mit dem Tonometer zu gering gefunden, was wahrscheinlich der höheren Wandspannung am Rande der Platte in diesen Fällen entspricht. Die dritte Reihe der Tabelle beweist auch wieder, dass der Druck in abgeschlossenen Ange durch die Anwendung des Tonometers beträchtlich steigt.

Beim normalen lebenden Kaninchen wird mit dem 
Tonometer ein Druck von $20 \mathrm{gr}$ gefunden; beim normalen menschlichen Auge ein Druck von ca. $17 \mathrm{gr}$. Messungen an pathologischen Augen sind noch nicht in genügender Anzahl ausgeführt worden, ich hoffe darüber später zu berichten.

Wie bei der Kritik des Fick'schen Tonometers hervorgehoben wurde, bestimmen wir nach dem neuen Princip nicht den wirklichen Druck im Auge, sondern die von verschiedenen Factoren abhängige Raumvergrösserung, welche bei Druck auf das Ange stattfinden kann. Ich möchte darum vorschlagen, den Tonus des Auges nicht mehr in Millimetern Quecksilber auszudrücken, sondern einfach in Grammen durch die Zahl, wie sie mit dem Tonometer gefunden wird.

Ich glaube, dass dieses neue Tonometer besonders am menschlichen Auge leichter zu appliciren ist als das Ficksche Instrument, und dass die damit gewonnenen Resultate constanter sein werden.

III.

\section{Bemerkungen über Ophthalmomanometrie.}

Wiewohl meiner Ansicht nach die Manometrie des Auges heutzutage einen gewissen Grad ron Vollkommenheit erreicht hat, so taucht doch zuweilen hier und dort die Meinung auf, dass man mit dem Manometer den Augendruck nicht annähernd genau bestimmen könne.

Auch Ostwalt beschreibt in seiner vorher citirten Arbeit einige misslungene Versuche, den intraocularen Druck manometrisch zu bestimmen, und wiewohl er nur die von Schultén angegebene Methode, nämlich die Einführung einer mit einem Manometer verbundenen Canüle in den Glaskörperraum versucht hat, so fühlt er sich berechtigt, zu behaupten, dass es "unumstösslich" feststeht, dass wir zur Zeit kein Mittel besitzen, um mit Hilfe eines Mano- 
meters "absolut genau" den Druck im Auge zu messen. Wenn Herr Ostwalt die Worte "absolut genan" wörtlich auffasst, so wird natiirlich keiner diesem widersprechen, weil allen unseren Wahrnehmungen Fehler auhaften; doch dass wir bis in Millimeter $\mathrm{Hg}$ den Augendruck manometrisch messen können, davon kann Jeder sich überzeugen, wemu er nur die richtigen Instrumente zur Verfügung hat mo eine gute Methode anwendet.

Vor Allem muss man den Druck nicht im Glaskörperraum bestimmen, sondern in der vorderen Kammer. Ich habe mich wiederholt überzeugt, dass die von Schultén(7) angegebene Canïle zur Einführung in den Glaskörper, nicht geeignet ist, eine freie Communication mit dem Inhalte des Bulbus herzustellen. Die von nir angewandte hatte genau die Form, und sogar noch ein etwas weiteres Lumen, wie die von Schultén beschriebene. Ich ïberzeugte mich durch Einführung eines entsprechenden Messingdrahtes, dass die Canüle überall denselben Durchmesser hatte. Fiuhrt man sie am ausgeschnittenen Thierauge neben dem Opticus oder in der Gegend des Corpus ciliare ein, so zeigt sich immer, dass wohl Flüssigkeit durch dieselbe aus dem Manometer ins Auge hineingetrieben werden kann, dass diese aber das Auge nicht mehr zu verlassen vermag, oder doch nur spurweise, weil der Glaskörper die Oeffunng abschliesst. Beim frischen Schweins- und Kaninchenauge nimmt man dasselbe wahr. Bei einem eben exstirpirten Bulbus eines. Kaninchens, wo die Canüle neben dem Opticus lag, stellte ich den Augendruck mittelst des Manometers erst auf $25 \mathrm{~mm} \mathrm{Hg}$, schloss dann das Auge ab, stellte den Druck im Manometer auf 0 und öffnete jetzt wieder die Communication mit dem Auge. Es blieb das Quecksilber dann genau stehen wie es stand, es trat also keine Spur von Flüssigkeit aus dem Auge aus. Derselbe Versuch wurde wiederholt mit einem Druck von $100 \mathrm{~mm} \mathrm{Hg}$, und es traten hierbei nur $5 \mathrm{~mm}$ Theilstriche Flüssigkeit aus, was einem 
Volum von 5 cbmm entspricht. Das Auge blieb steinhart, der Druck in $\mathrm{Hg}$ noch $80 \mathrm{~mm}$.

Der Glaskörper ist also nicht dünnflüssig genug, um durch eine Caniile ron $0,75 \mathrm{~mm}$ Oeffnung frei hindurch treten zu können. Bei gewissen Stellungen der Canüle tritt zuweilen auf einen Augenblick etwas bessere Communication ein, doch um für Messungen zu dienen, ist die Canüle nicht brauchbar. Dass die Oeffnung der Canüle frei im Glaskörper lag, davon überzeugte ich mich durch Abtragen des vorderen Theiles des Bulbus, während dieser in situ blieb. Auch war von Gerinnungen in der Canïle nicht die Rede, da bei kleinem Druck sofort grosse Tropfen die Oeffnung verliessen.

Dass Professor Schultén trotzdem mit einer Glaskörper-Canüle Druckmessungen hat austellen können, muss ich mir so erklären, dass er viel weitere Caniulen benützt, aber deren Maasse nicht richtig angegeben hat. Dickere Canülen als mit einem Lumen von $0,75 \mathrm{~mm}$ kann man aber nicht ins Auge einführen, ohne solche Störungen hervorzurufen, dass zuverlässige Versuche nicht möglich sind. Auch muss ich im Allgemeinen bemerken, dass eine Canïle, welche im Glaskörper liegt, unzweifelhaft mehr die normalen Ernährungsvorgänge und Druckverhältnisse im Auge stören muss, als eine, welche in die vordere Kammer eingeführt ist. Es ist sehr schön, wenn Professor Schultén an ein Manometer die Anforderung stellt, dass die Reizung der Cornea fortfallen muss, allein er muss keine stärkere Reizung des ${ }^{\circ}$ Anges dafür an die Stelle setzen. Und dass eine Durchbohrung von Sklera, Chorioidea und Retina in der Nähe des Corpus ciliare mit einer dicken Canüle, deren Spitze frei in Glaskörperraum beweglich ist, mehr Reizung des Auges hervorrufen muss, als eine nadelförmige Canïle, welche fest in der vorderen Kammer liegt, darüber kann meines Erachtens kein Zweifel bestehen. Von Reizerscheinungen, wie Schultén angiebt, habe ich nie etwas be- 
merken können. Wahrscheinlich wurde von Schultén eine zu dicke Canüle benützt. Die im hiesigen Laboratorium gebranchten Canülen haben einen äusseren Durchmesser von $0,7 \mathrm{~mm}$, nehmen also beinahe keinen Raum ein. Werden sie vor der Anwendung mit Schmirgelpapier abgerieben, so dass sie völlig glatt sind, so sind sie sehr leicht mittelst eines pincettenförmigen Nadelhalters einzufïhren und von Verlust von auch noch so wenig Kammerwasser ist dabei nie die Rede. Die Communication durch die freie Oeffnung bleibt Stunden lang eine vorzügliche, wenn man nur darauf achtet, während der Versuche kein Kammerwasser in der Canüle stehen zu lassen, weil sonst durch Fibrimausscheidung allmählich Verstopfung des Lumens der Canüle eintritt.

Von einigen Experimentatoren ist verlangt worden, dass als Beweis einer guten Conmunication Pulsbewegungen an der Quecksilbersäule zu sehen sein müssen. Ich kann dies nicht zugeben. Oft habe ich keine Pulsbewegung wahrgenommen bei Versuchen, wo eine ideale Communication mit dem Auge bestand. Vielmehr habe ich den Findruck bekommen, dass die Pulsbewegung des Quecksilbers erst damn und nur da auftritt, wo das Auge etwas gereizt war. Ebenso wenig finde ich es beweisend für freie Communication des Augeninhaltes mit dem Manometer, wenn bei einer in den Glaskörper eingeführten Canüle an dem Quecksilber Pulsschwankungen wahrgenommen werden können. Kleine Druckschwankungen pflanzen sich nämlich auch durch eine elastische Membran weiter, und als solche kann man die vor der Oeffnung der Canüle liegende Glaskörpersubstanz betrachten. Man kann auch beobachten, dass, wenn bei einem todten Auge ein sehr kräftiger Druck mit den Fingern auf die Sklera keine Fluissigkeit aus dem Auge hinans zu treiben vermag, doch ein sehr schwacher Druck mit dem Finger sich als eine kleine Bewegung der Quecksilbersäule kund giebt. 
Um zuverlässige Resultate bei der Ophthalmomanometrie zu erhalten, braucht man weiter ein zweckmässig eingerichtetes Manometer. H. Höltzke (8) gebührt das Verdienst, zuerst ein Instrument angegeben zu haben, womit man den Augenduck genau bestimmen konnte. Nur fehlen an seinem Manometer einige Einrichtungen, welche den Gebrauch wesentlich erleichtern und eine schnelle und genaue Ablesung des vorhandenen Druckes möglich machen. Unabhängig von Höltzke hat Schultén ein Manometer angegeben, welches ebenfalls bezweckt, den Druck kennen zu lernen, wenn weder Flüssigkeit aus dem Auge heraus noch hineingetreten ist; doch haften seinem Instrument mehrere Fehler an. Erstens hat er als eigentliches Manometer ein dickeres Glasrohr angewandt, und dies auf der einen Seite mit einem Capillarrohr verbunden. Dadurch entstehen auf einer Seite die Fehler, welche einem Capillarrohr immer anhaften, und diese werden nicht durch eine gleichwerthige Einrichtung auf der anderen Seite compensirt. Zweitens hat das weite Rohr den Nachtheil, dass, wenn der Druck nicht genau getroffen ist, ein beträchtliches Quantum Flüssigkeit das Auge verlassen oder in dasselbe hineintreten kann, weil eben diese Vorgänge in dem weiten Rohr auf die Höhe des Quecksilbers wenig oder gar keinen Einfluss ausüben. Drittens ist für ein Manometer eine Luftblase in einem Thermometerrohr als Indicator nicht geeignet. Ich habe mich überzeugt, dass sogar in einem Rohr von 0,5 mm Durchmesser das Wasser, besonders bei stossweiser Bewegung der Flüssigkeitssäule, an einer Luftblase von $30 \mathrm{~mm}$ Länge vorbeischlüpft, und zwar in beträchtlicher Menge. Weiter scheinen mir auch unnöthig viele Gummischläuche bei der Zusammensetzung des Instrumentes verwendet worden zu sein. Obwohl dicke Gummischläuche einen kleineren Fehler geben, so dürfen sie doch für genaue Versuche nicht in zu grosser Länge und Anzahl benutzt werden. Zuletzt muss ich noch bemerken, dass es gerade wie bei 
Höltzke's Instrument sehr unbequem ist, die Höhe der Wassersäulen immer mit in Berechnung bringen zu müssen. Der Hauptfehler bei Schultén's Verfahren ist aber, wie schon hervorgehoben wurde, der Gebrauch einer Glaskörpercanüle.

Alle diese Fehler sind von Leber bei der Construction seines Manometers vermieden worden. Man findet die Beschreibung seines Instrumentes in der Arbeit von G. Rindfleisch (9) in diesem Archiv. Ich muss nur dazu bemerken, dass man eine Klemme zur Abschliessung des Gummischlanches bei der Canüle nicht braucht, weil der $T-H a h n$ für alle $Z$ wecke vollständig genügt. Bei diesem Manometer wurde die Form des Höltzke'schen Instrumentes der Hauptsache nach beibehalten, weil gegen ein einfaches Manometer, mit einem Quecksilber-Reservoir unten, verschiedene technische Einwände zu machen waren. Weiter wurde oben an dem Manometerschenkel zur rechten Seite das kleine Wasserreservoir angebracht, wodurch das Wasserniveau an den beiden offenen Enden des Instrumentes inmer die gleiche Höhe hat. Dadurch wird die Ablesung sehr erleichtert. Man braucht nur die Höhe der Quecksilbersäule abzulesen und nachher für alle gefundenen Werthe die Correction anzubringen, dass für das specifische Gewicht des $\mathrm{Hg}$ nicht 13,5 sondern 12,5 in Rechnung gezogen wird. Es steht nämlich auf der linken Seite immer eine Wassersäule von der Höhe der $\mathrm{Hg}$ Säule, welche den Druck angiebt, mehr als auf der rechten Seite. Unentbehrlich ist weiter bei den meisten Versuchen die oberhalb des Hahnes angebrachte Spritze. Fehler durch Capillarwirkung bestehen bei diesem Instrument nicht, weil diese sich beiderseits in dem überall gleichweiten Rohr aufheben. Das Rohr hat iubrigens noch eine Weite, dass $1 \mathrm{~mm}$ Höhe 1 cbmm Inhalt entspricht.

Durch eine geänderte Eimrichtung ist es neuerdings gelungen, das Doppelmanometer entbehrlich zu machen und 
statt dessen ein einfaches Manometer mit Quecksilberreservoir zu benutzen, indem die Stopfbüchse neben dem Manometer aufgestellt und in ihrem unteren Theil mit Quecksilber, in ihrem oberen mit Wasser gefüllt wurde. Dadurch wird erreicht, dass der metallene Stempel und die übrigen Theile, welche aus Metall angefertigt werden müssen, nicht mit dem Quecksilber in Berührung kommen. Prof. Leber wollte sich ein Manometer eimrichten lassen, womit man zugleich genau den Augendruck bestimmen, und auch messen könnte, wie viel cbmm in der Zeiteinheit bei constantem Druck aus dem Auge filtrirten. Um das Letztere möglich zu machen, muss man entweder in einem calibrirten Rohr die Verschiebung der Flüssigkeitssäule ablesen können, oder im Stande sein, eine markirte Stelle in der Flüssigkeitssäule wieder nach ihrem Ausgangspunkte zurückzuführen, und die dafür eingepresste Menge Flüssigkeit zu messen. Um bei constantem Druck filtriren zu können, muss das Manometer so weit sein, dass das Steigen resp. Sinken des Quecksilbers verschwindend klein ist gegen die Höhe des angewandten Druckes.

Um eine Stelle in der Flüssigkeitssäule zu markiren, braucht man einen zuverlässigen Indicator, $d$. h. einen solchen, an dem die Elüssigkeit nicht vorbeischlüpft. Wir versuchten als Indicator in einem calibrirten Rohr: 1) eine Luftblase in einer Wassersäule; dieselbe giebt fast keinen Fehler bei langsamer, gleichmässiger Bewegung der Flüssigkeit; bei stossweiser Bewegung, wie sie bei Schliessung und Oeffnung eimes Hahnes auftritt, wenn der Druck auf beiden Seiten desselben verschieden ist, treten erhebliche Fehler auf; 2) einen Tropfen Oel oder Petroleum in einer Wassersäule; derselbe emulgirt sich sehr bald mit dem Wasser, und ist also unbrauchbar; 3) ein horizontales Rohr, welches theils mit Quecksilber, theils mit Wasser gefullt war; das Wasser dringt sogar in einem Rohr von $1 \mathrm{~mm}$ Durchmesser des Lumens noch oberhalb des Quecksilbers vor; 4) eine kleine 
aus Wachs und Siegellack angefertigte Kugel, welche dasselbe specifische Gewicht hatte wie Wasser und fast genau in das mit Wasser gefüllte Rohr passte; auch dies gab bei schnellerer Bewegung erhebliche Fehler. Wir glaubten dann eine befriedigende Methode darin gefunden zu haben, dass beim Anfang des Filtrationsversuches das Quecksilber auf der Seite des Auges gerade auf die Grenze eingestellt wird, wo ein dünnes Rohr in einen weiten Ballon übergeht. Während des Filtrirens verbreitet sich das Quecksilber dann auf dem Boden des weiten Gefässes, ohne dass der Druck merklich steigt; ist der Versuch beendet, so kann man mittelst einer Spritze das Quecksilber an seine frühere Stelle zurückdrängen und in dem dïnnen, calibrirten Schenkel des Manometers behufs der Messung aufsteigen lassen. Die Ausführung hat aber unseren Erwartungen nicht entsprochen, so dass für Filtrationsversuche, wo keine raschen Druckschwankungen vorkommen, doch eine Luftblase in einer horizontalen Wassersäule als Tndicator vorzuziehen sein diüfte.

\section{IV.}

Versuche über die Elasticität der Augenkapsel und über das Verhältniss von Volumszunahme und Formveränderung des Auges zu der Höhe des intraocularen Druckes.

Es schien mir nothwendig, diese vorstehenden Bemerkungen über Manometrie vorauszuschicken, ehe ich zur Mittheilung meiner über die Elasticität der Sklera gemachten Versuche übergehe. Es ist nämlich über diesen Gegenstand, soweit ich gesehen habe, noch sehr wenig gearbeitet worden; und gerade die wenigen Versuche, welche man in der Literatur antrifft, sind von Schultén mit seinem Manometer vorgenommen. Zwar hat Ad. Weber (10) schon viel früher die Elasticität von Streifen menschlicher 
Sklera zu bestimmen versucht, allein die Verhältnisse sind am intacten Bulbus, wo die Spannung die Sklera in allen Flächenrichtungen auszudehnen strebt, doch etwas andere.

Schultén (7) bestimmte am Hunde- und Kaninchenauge, wieviel Flüssigkeit bei einer gewissen Druckerhöhung in das Auge hineintrat. Dabei wurde der Druck jedesmal von $1 \mathrm{~mm} \mathrm{Hg}$ an gesteigert, und wieder auf 1 zurïckge. führt. Um den durch die Filtration verursachten Fehler zu eliminiren, nahm er den mittleren Werth von dem Volumen der Flüssigkeit, welches in das Auge eintrat, und demjenigen, welches wieder aus dem Bulbus zurückkehrte. Die Caniile des Manometers wurde in das, an dem Opticus aufgehängte Auge, neben ersterem in den Glaskörper eingeführt.

Auf die Einwände gegen die Glaskörpercanüle brauche ich nicht zurückzukommen. Ich kann nur noch hinzufügen, dass es sehr viel Kraft erfordert, um durch eine frei im Glaskörper liegende Canüle von 1,5 $\mathrm{mm}$ Durchmesser des Lumens den Inhalt eines frischen Schweinsanges auszupressen.

Was die Resultate der Versuche betrifft, so ist zu bemerken, dass Schultén kein Recht hat, die gefundene Volumzunahme bei jedem Druck als elastische Dehnbarkeit des Bulbus zu deuten. Bekanntlich haben die Augen von Hunden und Kaninchen keine reine Kugelgestalt, besonders nicht, wenn sie bei einem Druck von einem $m \mathrm{~m} \mathrm{Hg}$ an einem Punkte ihrer Oberfläche aufgehängt sind. Wir haben daher bei diesen Versuchen immer mit zwei Factoren zu rechnen, erstens mit der Gestaltsänderung des Bulbus weil dieser danach strebt, der Kugelgestalt sich zu nähern, und zweitens mit der elastischen Ausdehnung der Augenhüllen. Der erste wird sich besonders bei den niedrigeren Druckstufen geltend machen, der zweite bei den höheren, und es wird von dem Verhältniss zwischen diesen beiden 
Factoren abhängen, ob sie in einer Curve der Volumzunahme gesondert sichtbar werden können.

Um zu untersuchen, in wie weit die Elasticität der Augenhüllen und die Formveränderung des Bulbus an der Volumzunahme des letzteren bei einer gewissen Druckerhöhung theilnehmen, habe ich versucht, äusserlich bei Kaninchen- and Schweinsaugen den Durchmesser des Auges in drei senkrecht aufeinander stehenden Richtungen bei verschiedenen Druckhöhen zu messen. Ich bediente mich dabei folgender Instrumente.

Um das enucleirte Kaninchenauge zu messen, wurde nebenstehender Tasterzirkel benuitzt; die Abbildung, in halber wirklicher Grösse hergestellt, macht eine ausfuhrliche Beschreibung unnöthig. An den Enden $a$ und $b$ befinden sich zwei feine Spitzen, welche es möglich machen, während des Versuches ge-

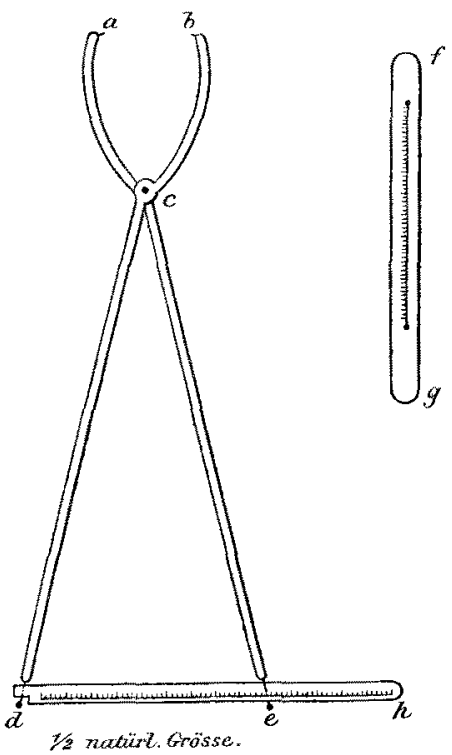

Fig. 6. nau dieselben Stellen zu behalten. Die Branchen $b d$ und ae drehen sich um die Axe $c$. Ein kleiner Maassstab $d h$ liegt bei $e$ zwischen zwei glatten Nadeln, und ist bei $d$ um eine Axe drehbar. Das Ganze ist aus leichtem, harten $\mathrm{Holz}$ angefertigt, und die Bewegungen in den Punkten $c, d$ und $e$ finden mit sehr wenig Reibung statt. Um die Distanz des Bulbus bis zur Axe $c$ messen zu können, kann man noch einen kleinen Maassstab $f g$ einlegen, der durch eine linienförmige Oeffnung in der Mitte 
um eine in der Axe $c$ stehende kleine Nadel frei verschiebbar ist.

Um beim lebenden Kaninchenauge den verticalen und horizontalen Aequatorialdurchmesser zu messen, kann man dasselbe Instrument benutzen. Für den Durchmesser von der Cornea oder von dem Limbus bis zur Opticusgegend gebrauchte ich nebenstehendes Instrument. Das Princip ist dasselbe. Die eine Branche

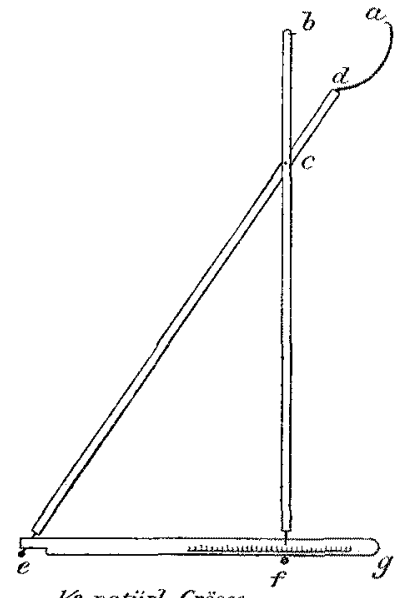

1/2 natürl Grösse

Fig. 7. ist hier aber gerade, die andere führt an ihrem Ende eine gebogene Nadel $a d$, welche hinter dem Auge vorgeschoben werden kann, wenn die Muskeln durchtrennt sind. Die Maasse sind so gewählt, dass, wenn $a b$ ungefähr dem Durchmesser des Auges entspricht, bf ungefähr senkrecht auf dem Maassstabe $g e$ steht. $g e$ ist in diesem Stande auch ungefähr parallel $a b$. Die Verschiebung von $f$ ist dann fast genau der Grössenzunahme des Durchmessers proportional. Zur constanten Fixirung ein und desselben Punktes ist in $b$ auch wieder eine schiefgestellte Nadelspitze angebracht.

Um ganz geringe Längenzunahmen des Durchmessers zu messen, habe ich folgende Einrichtung getroffen. An das eine Ende eines $30 \mathrm{~cm}$ langen Thermometerrohrs von sehr engem Lumen ist eine trichterförmige Erweiterung von 2,5 cm Durchmesser angeblasen. Auf die Trichteröffnung wird eine Gummimembran befestigt, jedoch ohne dieselbe wesentlich zu spannen. Auf die elastische Membran wird in der Mitte ein Scheibchen Carton von $1 \mathrm{~cm}$ Durchmesser geklebt, welches in seinem Centrum eine stumpfspitzige 
Nadel von $1 \mathrm{~cm}$ Länge trägt. Der Trichter wird mit gefärbter Flüssigkeit soweit gefüllt, dass im Anfang des Thermometerrohres ebenfalls eine kleine Flüssigkeitssäule steht. Eine kleine Verschiebung der Nadel nach innen wird an diesem Instrument durch eine sehr grosse Verschiebung der Flüssigkeitssäule im dümnen Rohr angezeigt. Die Theilung auf dem Rohr muss man mit einer geeigneten Einrichtung mittelst Mikrometerschraube herstellen. Bei den von mir benutzten Apparaten entspricht $1 \mathrm{~mm}$ Verschiebung der Nadel nach innen ungefähr $180 \mathrm{~mm}$ Verschiebung der Fliissigkeit im Thermometerrohr.

Die Anwendung dieses Messtrichters geschieht auf folgende Weise. Bei dem zu untersuchenden Auge wird die Nadel des Manometerrohres in die vordere Kammer eingeführt; der Bulbus wird dann, mit der Cornea nach oben, auf drei stumpfe Spitzen, welche unweit von einander senkrecht in einem Brett befestigt sind, gelegt, derart, dass der Sehnerv zwischen den drei Spitzen steht. Der Druck im Bulbus wird dann niedrig gestellt, z. B. anf $5 \mathrm{~mm} \mathrm{Hg}$. Um jetzt den verticalen Durchmesser des Auges zu messen, wird von der einen Seite der Stift eines Messtrichters, der auf demselben Brett befestigt ist, genau gegen die Skleru gestellt, ohne dass derselbe auch nur den geringsten Eindruck in den Bulbus macht. Auf der entgegengesetzten Seite ist ein unbeweglicher Stift angebracht, der auch nur eben das Auge beruihrt. Der horizontale Durchmesser des Auges wird zur selben Zeit auf dieselbe Weise zwischen einen Messtrichter und eine feste Spitze gelegt. Um den Durchmesser vom Centrum der Cornea bis zum Opticus zu messen, wird auch die Cornea noch mit der Spitze eines Trichters in Berührung gebracht. Das Rohr desselben ist aber dreinal rechtwinklig umgebogen, wodurch der Theil, der zum Messen benutzt wird, wieder horizontal verläuft und zwar in derselben Höhe wie das Niveau der elastischen Membran.

Wenn die Membranen der Trichter sehr wenig Spanv. Graefe's Archir für Ophthalnologie. XLI. 2. 
nung haben, kann man die Apparate etwas weiter vorschieben, so dass die Membranen durch Druck auf die Skleraoberfläche eine Spur eingedrückt werden. Dies kann geschehen, ohne dass ein Eindruck in den Bulbus hervorgerufen wird, weil es die Rigidität der Sklera verhütet. Diese Anordnung ergiebt den Vortheil, dass auch eine Verkürzung des Durchmessers durch Verschiebung der Flüssigkeit angedeutet wird.

Ich habe diese Glastrichter bis jetzt nur bei frischen Schweinsaugen benutzt; diese Augen zeigten nämlich eine sehr ins Auge springende Zunahme des optico-coruealen Durchmessers, während mit dem Messzirkel absolut keine Aenderung der aequatorialen Durchmesser nachzuweisen war. Es kam mir dabei darauf an, zu sehen, ob eine Verkürzung dieser Durchmesser vielleicht erfolgte während der Volumszunahme und dafür wurden die empfindlichen Messtrichter angefertigt. Wenn ich später Gelegenheit habe, normale menschliche Augen auf die Ausdehnung der Sklera zu untersuchen, so werde ich noch viel grössere Trichter benutzen, um die Bewegung der elastischen Membran möglichst ohne Widerstand erfolgen zu lassen. Die Empfindlichkeit des Apparates wird dadurch natürlich auch noch erhöht.

Bei den Versuchen mit den Zirkeln wurde auch möglichst darauf geachtet, dass die Spitzen keinen Eindruck in die Sklera hervorriefen. Man würde sonst natürlich zu ganz falschen Resultaten kommen, da bei Druckerhöhung der Eindruck verschwinden, und dies sich jetzt als Längenzunahme des betreffenden Durchmessers kundgeben würde.

Nach dieser Auseinandersetzung kann ich zur Mittheilung der Resultate meiner Messungen übergehen.

Die nebenstehende Tabelle giebt die an einem Schweinsauge gewonnenen Resultate. Andere Augen ergaben ähn- 


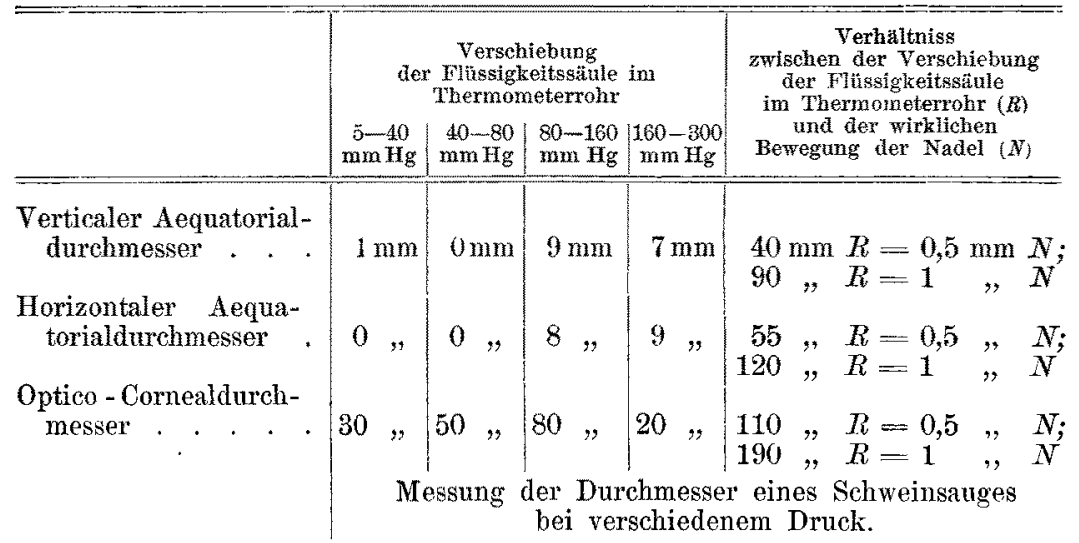

liche Resultate. Ich finde es aber genauer, hier keine Mittelwerthe anzugeben, weil dadurch eigenthïmliche Erscheinungen verborgen werden könnten. Ich komme darauf später zurück.

Man sieht also, dass bei dem Schweinsauge fast nux der optico-corneale Durchmesser zunimmt und zwar reell um ungefähr $1 \mathrm{~mm}$. Die anderen Durchmesser bleiben bis zu einem Druck von $80 \mathrm{~mm} \mathrm{Hg}$ constant, wenn wir die Verschiebung von $1 \mathrm{~mm}$ im Thermometerrohr bei der Messung des verticalen Aequatorialdurchmessers vernachlässigen. Hieraus geht hervor, dass die äquatorialen Durchmesser durch Ausdehnung der elastischen Augenhüllen ebensoviel an Länge zunehmen, als sie durch Annäherung des Bulbus an die Kugelgestalt abnehmen. Nur bei selr hohen Druckstufen übertrifft der erste Werth den letzteren an Grösse. Wie zu erwarten war, wird endlich eine Druckhöhe erreicht, wobei die Form des Bulbus der Kugelgestalt möglichst ähnlich ist, in unserem Fall bei $160 \mathrm{~mm}$ Hg. Bei höherem Druck kann Volumzunahme nur erfolgen durch Ausdehnung der Wand, und zwar jetzt in allen Richtungen, wie wir auch in der Tabelle sehen. 
Beim lebenden Kaninchen wurden der horiz. AequatorialDurchmesser, der horiz. Durchmesser am Cornearande, der optico-corneale Durchmesser, und der Abstand vom nasalen Cornearand bis zur temporalen Opticusseite gemessen. Die geraden Augenmuskeln wurden sammt der ganzen Conjunctiva erst vom Bulbus lospräparirt, wobei die Venae vorticosae geschont wurden. Auch einige Fasern vom Retractor wurden durchtrennt. Ich fand folgende Werthe:

\begin{tabular}{|c|c|c|c|c|c|c|}
\hline \multirow[t]{2}{*}{$\begin{array}{c}\text { Bei einem } \\
\text { intraocularen } \\
\text { Druck von }\end{array}$} & \multicolumn{2}{|c|}{$\begin{array}{l}\text { Horizontaler } \\
\text { Aequatorialdurch- } \\
\text { messer }\end{array}$} & \multicolumn{2}{|c|}{$\begin{array}{l}\text { Horizontaler } \\
\text { Durchmesser am } \\
\text { Cornearand }\end{array}$} & \multirow{2}{*}{$\begin{array}{c}\text { Nasaler } \\
\text { Cornearand } \\
\text { bis temp. } \\
\text { Seite des } \\
\text { Nerv, opt. } \\
\text { Nr. } 3\end{array}$} & \multirow{2}{*}{$\begin{array}{l}\text { Centrum der } \\
\text { Cornea bis } \\
\text { temp. Seite } \\
\text { des Nerv } \\
\text { opticus } \\
\text { Nr. } 3\end{array}$} \\
\hline & Nr. 1 & Nr. 2 & Nr. 1 & Nr. 2 & & \\
\hline 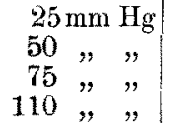 & $\begin{array}{l}15,5 \mathrm{~mm} \\
15,9 \% \\
16,3 \% \\
16,7 \%\end{array}$ & $\begin{array}{ll}15,75 & \mathrm{~mm} \\
15,9 & " \\
16 & " \\
16,7 & "\end{array}$ & $\begin{array}{l}13,7 \mathrm{~mm} \\
13,7 \quad " \\
13,7 \quad " \\
13,7 \quad "\end{array}$ & $\begin{array}{l}13,4 \mathrm{~mm} \\
13,6 " \\
13,6 ", \\
13,6 "\end{array}$ & $13,1 \mathrm{~mm}$ & $17,25 \mathrm{mn}$ \\
\hline
\end{tabular}

Mit dem kleinen Maassstab konnte ich keine Aenderung des Abstandes von der Axe des Apparates zum Scheitel der Cornea nachweisen weder beim Messen des äquatorialen Durchmessers, noch beim Messen des Durchmessers des Auges am Limbus corneae. Um nachzuweisen, ob bei der Ausdehnung die Krümmung der Cornea sich änderte, wurden die Krümmungsradien bei einigen Augen mit dem Ophthalmometer ron Javal bestimmt. Bei einem Versuch mit einer Glaskörpercanuile wurde der Radius gleich $6.683 \mathrm{~mm}$ gefunden bei einem Druck von $30 \mathrm{~mm} \mathrm{Hg}$, und gleich $6.726 \mathrm{~mm}$ bei einem Druck von $160 \mathrm{~mm} \mathrm{Hg}$. Der Versuch konnte dann nicht mehr wiederholt werden, weil die eingepresste Flüssigkeit das Auge nicht mehr durch die Glaskörpercanüle verlassen konnte. Vor und nach dem Durchschneiden der Recti war der Radius $=6.683 \mathrm{~mm}$.

Bei einem zweiten Auge wurde die Canüle vertical durch die vordere Kammer gefuihrt. Der horizontale Krüm- 
mungsradius wurde gemessen, und sowohl bei einem Druck von 30 als von $120 \mathrm{~mm} \mathrm{Hg}=5.959 \mathrm{~mm}$ gefunden. Es zeigte sich hier auch, dass durch die Einführung der nadelförmigen Canüle die Krümmung der Cornea nur eine Spur geändert war. Bei einem dritten Auge, wo ebenfalls die Caniule vertical in der vorderen Kammer lag, änderte sich der Radius bei einer Druckerhöhung von 30 auf $120 \mathrm{~mm}$ $\mathrm{Hg}$ von $6.285 \mathrm{~mm}$ in $6.209 \mathrm{~mm}$.

Weil, wie wir aus der Tabelle sehen, der Durchmesser des Auges am Cornearande bei Ausdehnung dieser Bulbi constant blieb, so dürfen wir aus den fast constanten Maassen des Cornearadius schliessen, dass die Cornea sich ebenfalls nicht messbar ausgedehnt hat.

Von W. Eissen (11) sind über die Hornhautkrümmung bei Drucksteigerung sehr ausfihhrliche Versuche angestellt worden; als Versuchsthier wurde das Kaninchen benützt; die Resultate waren sehr verschieden. Druckerhöhungen um $25 \mathrm{~mm} \mathrm{Hg}$ wurden aber meistens nicht ohne Gestaltveränderung ertragen. Jedenfalls aber wurden die Unterschiede in der Länge des Radius auch von diesem Experimentator sehr gering gefunden.

Die älteren Versuche von Schelske (14) uiber denselben Gegenstand wurden an enucleirten Angen angestellt. Er fand, dass bei Drucksteigerung von 0 bis $150 \mathrm{~mm} \mathrm{Hg}$ die Krümmung der Cornea zuweilen stärker, bei Drucksteigerung von 150 bis $250 \mathrm{~mm} \mathrm{Hg}$ meist danernd wieder geringer wurde. Die Vergrösserung des Radius war sehr verschieden, meist aber sehr gering.

Bei dem eben exstirpirten Kaninchenauge kömmen begreiflicherweise die Skleramaasse genauer gemessen werden, besonders der optico-corneale und der verticale Aequatorial-Durchmesser. Ich erhielt bei einem Auge, wo zuerst schnell die Volumzunahme bei verschiedenem Druck gemessen war, folgende Werthe. 
W. Koster.

\begin{tabular}{|c|c|c|c|c|c|c|}
\hline $\begin{array}{l}\text { Bei einem } \\
\text { intraocularen } \\
\text { Druck von }\end{array}$ & $\begin{array}{c}\text { Horizon- } \\
\text { taler } \\
\text { Aequa- } \\
\text { torial- } \\
\text { Durch- } \\
\text { messer }\end{array}$ & $\begin{array}{c}\text { Verticaler } \\
\text { Aequa- } \\
\text { torial- } \\
\text { Durch- } \\
\text { messer }\end{array}$ & $\begin{array}{l}\text { Durch- } \\
\text { messer vom } \\
\text { Centrum der } \\
\text { Cornea zur } \\
\text { Opticus- } \\
\text { gegend tem- } \\
\text { poral }\end{array}$ & $\begin{array}{l}\text { Durch- } \\
\text { messer vom } \\
\text { nasalen } \\
\text { Cornea- } \\
\text { rande zur } \\
\text { Opticus- } \\
\text { gegend } \\
\text { temporal }\end{array}$ & $\begin{array}{l}\text { Durch- } \\
\text { messex am } \\
\text { Cornea- } \\
\text { rand: } \\
\text { vertical }\end{array}$ & $\begin{array}{l}\text { Durch- } \\
\text { messer am } \\
\text { Cormea- } \\
\text { rand: } \\
\text { horizontal }\end{array}$ \\
\hline $\begin{array}{r}5 \mathrm{mmHg} \\
25 \quad " \quad " \\
100 " \# " \\
160 " y\end{array}$ & $\begin{array}{l}19 \mathrm{~mm} \\
19,3 \% \\
19,6 \% \\
19,6 \%\end{array}$ & $\begin{array}{l}18,4 \mathrm{~mm} \\
18,6, \\
18,7 \\
18,7\end{array}$ & $\begin{array}{l}16,25 \mathrm{~mm} \\
16,9 ", \\
17,1 " \text { " } \\
17,2 " ~\end{array}$ & $\begin{array}{l}16,7 \mathrm{~mm} \\
17,4, \\
17,6 \% \\
17,7,\end{array}$ & $\begin{array}{l}13,5 \mathrm{~mm} \\
13,5 " \\
13,5 " \\
13,5 "\end{array}$ & $\begin{array}{l}11,7 \mathrm{~mm} \\
11,7 \% \\
11,7 " \\
11,7 \%\end{array}$ \\
\hline
\end{tabular}

Ausdehnung der Sklera beim exstirpirten Kaninchenauge.

Wenn wir diese Werthe vergleichen mit denen, welche beim lebenden Kaninchen gefunden wurden, so fällt uns sofort der Unterschied auf, dass beim letzteren die äquatorialen Durchmesser bei einer Drucksteigerung über $25 \mathrm{~mm}$ $\mathrm{Hg}$ noch bedeutend an Länge zugenommen haben, während beim todten Auge bei derselben Drucksteigerung diese Durchmesser fast unverändert blieben, oder nur sehr geringe Veränderung zeigten. Auf der anderen Seite ändert sich der optico-comeale Durchmesser beim lebenden Auge fast nicht, während das exstirpirte Auge in dieser Richtung bedeutend an Länge zunimmt. Dieselbe Erscheinung haben wir beim Schweinsauge wahrgenommen.

Die Maasse beim lebenden Thier, wiewohl an verschiedenen Augen gewonnen, legten die Vermuthung nahe, dass der enucleirte Bulbus eine andere Form haben könnte als das Auge in der Orbita beim lebenden Thiere. Ich habe darum an vier Augen Messungen angestellt, um diesen Punkt zu entscheiden.

Wenı wir die Tabelle aut der folgenden Seite näher betrachten, so sehen wir, dass die Form des Bulbus sich nicht wesentlich ändert bei der Durchschneidung der Muskelu und des N. opticus während des Lebens und ebensowenig durch Aufhören des Blutdruckes in den Grefässen. Theoretisch genommen miissen diese Faktoren einen Einfluss haben, 
Beiträge zur Tonometrie und Manometrie des Auges.

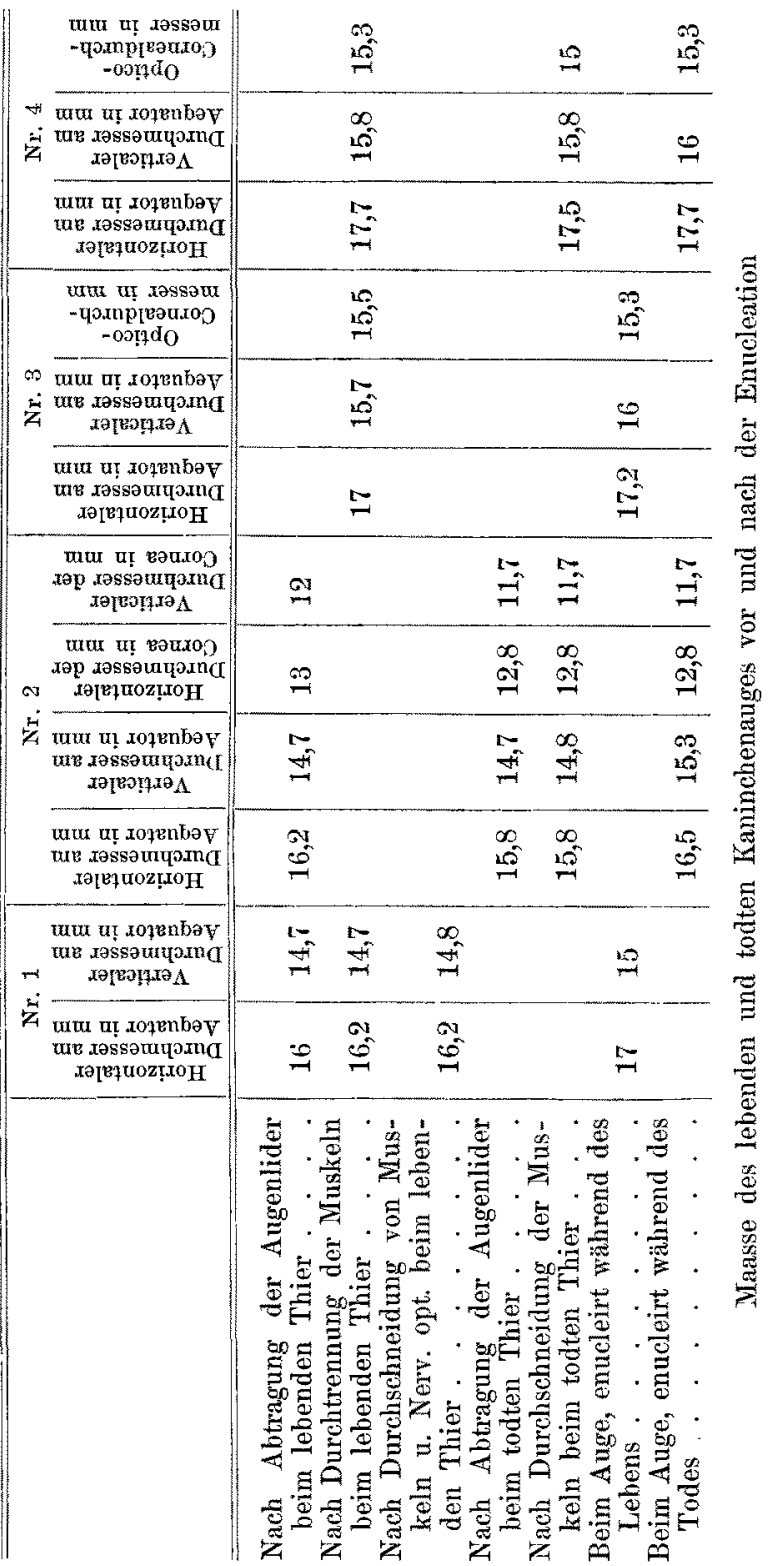


doch praktisch zeigt es sich, dass die Form der Sklera an und für sich fest geung ist, um deren Einwirkung Widerstand zu leisten. Die kleinen Unterschiede bei den Maassen müssen als Fehler angesehen werden; es wurde hier natürlich jedesmal der kleine Messzirkel abgenommen und in einem anderen Durchmesser wieder angelegt. Bei $\mathrm{Nr} .2$ wurden nicht nur die Augenlider abgetragen, sondern auch der Orbitalrand mit einer starken Scheere theilweise resecirt, um beim ruhenden Bulbus die Messung anstellen zu können. Bei 1,3 und 4 wurde der Bulbus zuweilen eine Spur aus der Orbita hervorgezogen, um das Instrumentchen anlegen zu können.

Aus diesen letzten Messungen geht auch hervor, dass wir die Messungen am enucleirten Kaninchenauge, welche selbstverständlich immer genauer sind als am Auge in der Orbita, ohne erheblichen Fehler auf das lebende Auge iihertragen können.

Wir müssen jetzt noch verfolgen, wie die Volumzunahme des Bulbus sich bei den verschiedenen Druckstufen verhält. Ich habe hier auch wieder mit dem Schweinsund Kaninchenauge experimentirt. In der folgenden Tabelle sind die Resultate zusammengestellt. (s. S. 153.)

Wie aus der Tabelle ersichtlich ist, sind die Versuche so angestellt, dass eine bekannte Quantität Flüssigkeit ins Auge hineingepresst, und dann der dabei entstehende Druck abgelesen wurde. Es wurde angefangen mit einem Druck von $1 \mathrm{~mm}$ Hg. In höchstens 5 Secunden war jedesmal das bestimmte Quantum mit der Stempelschraube des Manometers in das Auge eingepresst, und der Druck bestimmt. Der Druck wurde dann wieder auf $1 \mathrm{~mm}$ zurückgestellt, und eine weitere Bestimmung gemacht. Jedesmal wo zwischen den Volumzahlen ein Strich steht, wurde von einem höheren Druck ausgegangen, so resp. von 40, 80, 110 u. s. w. Die kleine Quantität Flüssigkeit, welche in den 5 Secunden aus dem Auge filtrirte, kann vernachlässigt 
Beiträge zur Tonometrie und Manometrie des Auges.

\begin{tabular}{|c|c|c|c|c|c|}
\hline \multicolumn{3}{|c|}{ Schweinsauge } & \multicolumn{3}{|c|}{ Kaninchenange } \\
\hline $\begin{array}{l}\text { Volum- } \\
\text { zunahme } \\
\text { in cbmm } \\
\text { von }\end{array}$ & $\begin{array}{l}\text { Druck- } \\
\text { steigerung } \\
\text { in mm Hg } \\
\text { von }\end{array}$ & $\begin{array}{l}\text { Volum- } \\
\text { zunahme } \\
\text { pro } \\
\text { mn Hg } \\
\text { in ebma }\end{array}$ & $\begin{array}{l}\text { Volum- } \\
\text { zunahme } \\
\text { in cbmm } \\
\text { von }\end{array}$ & $\begin{array}{l}\text { Druek- } \\
\text { steigerung } \\
\text { in } \underset{\text { pon }}{\mathrm{mg}} \\
\text { pon }\end{array}$ & $\begin{array}{c}\text { Volum } \\
\text { zunahme } \\
\text { pro } \\
\text { mm Hy } \\
\text { in chmm }\end{array}$ \\
\hline $0-10$ & $1-1,5$ & 20 & $0-10$ & $1-1,5$ & 20 \\
\hline $10-20$ & $1,5-2,5$ & 10 & $10--20$ & $1,5-5$ & 2,9 \\
\hline $20-30$ & $2,5-3,5$ & 10 & $20-30$ & $5-6,5$ & 6 \\
\hline $30-40$ & $3,5-4,5$ & 10 & $30-40$ & $6,5-8$ & $b$ \\
\hline $40-50$ & $4,5-6$ & 6 & $40-50$ & $8-11$ & 3 \\
\hline $50-60$ & $6-7,5$ & 6 & $50-60$ & $11-16$ & 2 \\
\hline $60-70$ & $7,5-9,5$ & 5 & $60-70$ & $16-18$ & 5 \\
\hline $70-80$ & $9,5-12$ & & & & \\
\hline $80-90$ & $12-14,5$ & 4 & $70-75$ & $18-22$ & 1,25 \\
\hline $90-100$ & $14,5-16$ & 6 & $75-80$ & $22-30$ & 0,63 \\
\hline $100--110$ & $16-19$ & 3 & $80-85$ & $30--36$ & 0,83 \\
\hline $110-115$ & $19-22$ & 1 & $85-90$ & $36-\mathbf{4}$ & 0,63 \\
\hline $115-120$ & $22-24$ & 2,5 & $90--95$ & $44-58$ & 0,36 \\
\hline $120-125$ & $24-26,5$ & 2 & $95-100$ & $58-70$ & 0,42 \\
\hline $125-130$ & $26,5-28,5$ & 2,5 & $100-105$ & $70-80$ & 0,5 \\
\hline $130-135$ & $28,5-32$ & 1,4 & $105-110$ & $80-95$ & \\
\hline $135-140$ & $32-36$ & 1,25 & $110-115$ & $95-117$ & 0,23 \\
\hline $140-145$ & $36-40$ & 1,25 & $115-120$ & $117-131$ & \\
\hline $145-150$ & $40-46$ & 0,83 & $120-125$ & $131-158$ & 0,18 \\
\hline $\begin{array}{l}150-155 \\
155-160\end{array}$ & $\begin{array}{l}46-49 \\
49--55\end{array}$ & $\begin{array}{l}1 \\
0,83\end{array}$ & & & \\
\hline $160-165$ & $55-61$ & 0,83 & & & \\
\hline $165-170$ & $61-71$ & 0,5 & & & \\
\hline $170-175$ & $71-78$ & 0,7 & & & \\
\hline $175-180$ & $78-84$ & 0,8 & & & \\
\hline $180-185$ & $84-98$ & 0,4 & & & \\
\hline $185-190$ & $98-107$ & 0,5 & & & \\
\hline
\end{tabular}

Volumzunahme eines Schweins - und Kaninchenauges bei verschiedenem Druck.

werden. Ich habe hier nur zwei von den Versuchen mitgetheilt und keine Mittelwerthe aus den angestellten Versuchen gezogen, weil bei der Volumzunahme constant einige Unregelmässigkeiten vorkommen, welche meines Erachtens nicht als Fehler aufgefasst werden können. Am besten 
zeigt sich dies, wenn wir die gefundenen Werthe in einer Curve aufzeichnen. Die Linie, welche in nebenstehender Fig. 8 die Volumzunahme des Schweinsauges in cbmm pro $\mathrm{mm} \mathrm{Hg}$ Drucksteigerung angiebt, sinkt erst sehr schnell nach unten, um bei $13 \mathrm{~mm} \mathrm{Hg}$ plötzlich wieder zu steigen. Solche Hebungen deuten nach meiner Meinung auf einen

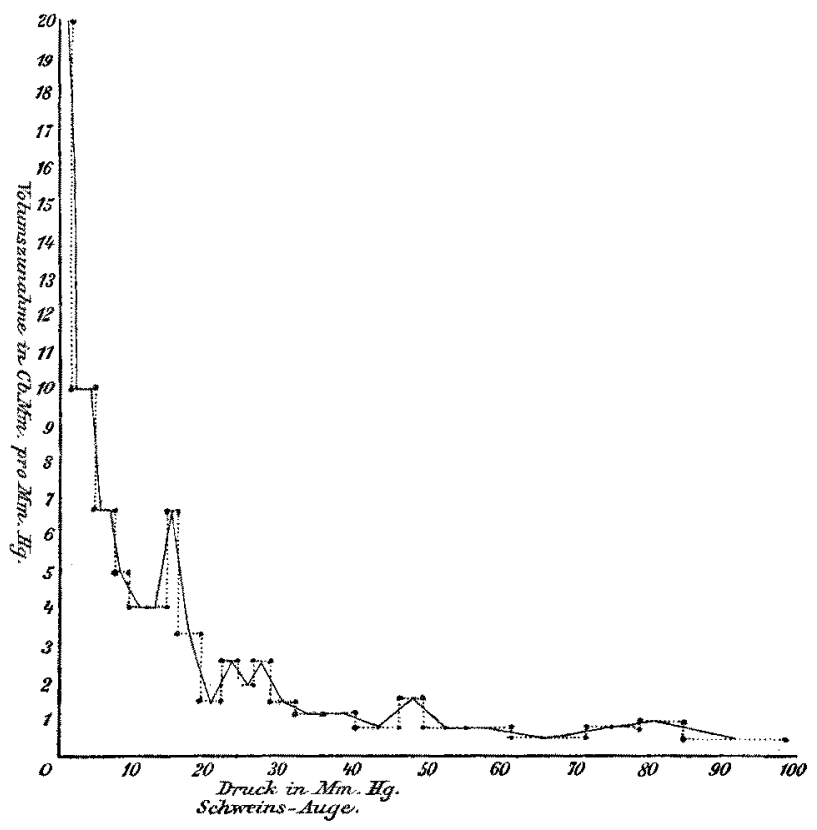

Fig. 8 .

plötzlich überwundenen Widerstand hin, denn bei demselben Bulbus sah ich diese Unregelmässigkeit fast genau an derselben Stelle der Curve wieder auftreten, wenn die ganze Bestimmung nach Ablauf der ersten wiederholt wurde.

An den anderen Curven (Fig. 9), welche die totale Volumzunahme darstellen, sehen wir erstens auch den Ausdruck der genannten Unregelmässigkeiten, und zweitens, dass die Volumzunahme bei einer Drucksteigerung von 1 bis 
Beiträge zur Tonometrie und Manometrie des Auges. 155

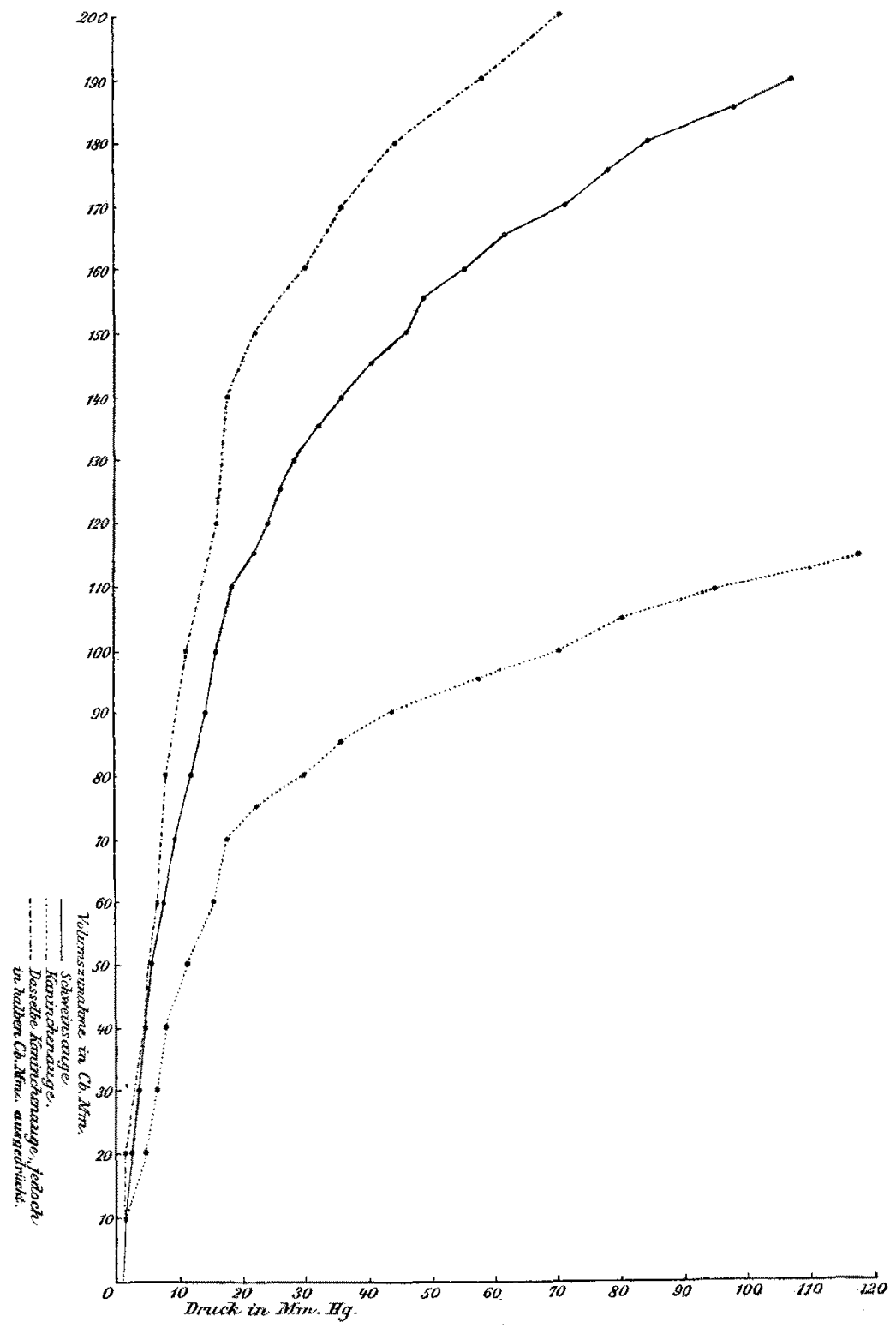

Fig. 9. 
20 bis $30 \mathrm{~mm} \mathrm{Hg}$ am stärksten ist und proportional der Druckhöhe, um allmählich abzunehmen bis zu einer Druckhöhe von 40 bis $50 \mathrm{~mm} \mathrm{Hg}$, wo sie wieder anfängt, der Drucksteigerung proportional zn werden. Wenn wir jetzt bedenken, dass die Veränderung der Augendurchmesser sich ebenfalls bei den niedrigen Druckstufen am meisten zeigte, und zwar vorwiegend in einer Axe, während bei höherem Druck die Ausdehnung des Bulbus kaum messhar war, so liegt der Schluss auf der Hand, dass die anfängliche beträchtliche Volumzunahme hauptsächlich der Formveränderung des Bulbus zuzuschreiben ist, welcher danach strebt, die Kugelgestalt anzunehmen, während die kleinere Volumzunalıme bei den höheren Druckstufen wirklich der Ausdehnung der Augenhüllen zuzuschreiben ist. Es steht auch ganz im Einklang mit den bekannten Gesetzen der Elasticität, dass bei mässiger Belastung, d. h. hier bei nicht ungewöhnlich hoher Drucksteigerung, die Ausdehnung der Druckhöhe proportional ist.

Ein weiterer Beweis dafür, dass die anfängliche starke Volumzunahme der Formveränderung zugeschrieben werden kann, zeigen die Curven vom Schweins- und Kaninchenauge wenn man sie für denselben Inhalt berechnet. Das Kaninchenauge hat einen Inhalt von ca. $3000 \mathrm{cbmm}$, das Schweinsauge von ca. $6000 \mathrm{cbmm}$ bei $0 \mathrm{~mm} \mathrm{Hg}$. Wenn wir also die beim Kaninchenauge gefundenen Werthe verdoppeln und die Curve zeichnen, so können wir diese mit der vom Schweinsauge vergleichen, weil bei beiden der Ausgangspunkt dann ca. $6000 \mathrm{cbmm}$ ist. Wir sehen daraus, dass die beiden Curven im Anfang fast denselben Weg nehmen; dies weist darauf hin, dass hier nur Formveränderung im Spiel ist. Nachher übersteigt die Curve rom Kaninchenange die vom Schweinsauge beträchtlich an Höhe, was damit stimmt, dass die dünnere Kaninchensklera durch einen bestimmten Druck relativ viel mehr ausgedehnt wird.

Bei den Versuchen über die Volumzunahme wurde der 
Bulbus an der nadelförmigen Canüle, welche in die fordere Kammer eingeführt war, aufgehängt. Ich habe mich uiberzeugt, dass bei dieser Versuchsanordnung die Schwere des Bulbus keinen Einfluss hat auf die Form der Sklera. Wenn ich bei ein und demselben Auge zuerst die Bestimmung machte, während das Auge in der Luft hing, und nachher, wenn der Bulbus in Kochsalzlösung von $3 / 4 \%$ untergetaucht war, wobei also die Schwere keinen merklichen Einfluss haben konnte, so bekam ich dasselbe Resultat; die Curven dieser Messungen deckten einander fast vollständig.

Ich beanspruche nicht, in diesem Aufsatz auch nur einigermassen den Gegenstand der Ausdehnung der Augenhüllen und der Formveränderung des Bulbus erschöpft zu haben. Es bestehen aber über diese Vorgänge noch so unklare Ansichten, und seit den Arbeiten Schultén's $(7,12)$ hat man so oft die beiden zusammengeworfen, dass es mir erwünscht schien, schon jetzt die Resultate dieser Versuche hier mitzutheilen.

\section{Literatur.}

1) A. Fick, Teber Messung des Druckes in Auge. Arch. f. die ges. Phys. Bd. XLII.

Rud. Arm. Fick, Ein neues Ophthalmotonometer. Inaugural-Dissertation. Wiurzburg, 1888.

2) Maklakoff, L'Ophthalmotonometrie. Arch. d'Ophthalmologie $\mathrm{V}, 1885$.

3) F. Ostwalt, Ophthalmotonometriselie Studie, v. Graefe's Arch. Bd. XL. Th. 5. 1894.

4) A. Weber, Zeliender's klin. Monatsbl. VI. 1868. S. 405.

5) Handb. der Augenh. von Graefe u. Saemisch. Tl. III. S. 190, Snellen u. Landolt, Ophthalmotonometrie.

6) Imbert, Théorie des Ophthalmotonomètres, Archives d'Ophthalmologie. 1885. V.

7) M. W. r. Schultén, Experimentelle Untersuchungen über die Circulationsverlältnisse des Auges, $v$. Graefe's Arch. XXX. 3. 1894, p. 6. 
158 W. Koster. Beiträge zur Tonometrie u. Manometrie d. Auges.

8) H. Höltzke, Experimentelle Untersuchungen über den Druck in der Augenkammer, v. Graefe's Arch. XXIX. 2. S. 5. 1883.

9) G. Rindfleisch, Experim. Unters. über die bei der eitrigen Chorioiditis auftretende Herabsetzung des intraoeularen Druckes. v. Graefe's Arch. XXXVIII. 2. S. 221. 1892.

10) Ad. Weber, Die Ursache des Glaukoms, v. Graefe's Arch. XXIII. 1. S. 23.1887.

11) W. Eissen, v. Graefe's Archiv. XXXIV. 2. S. 64. 1888.

12) M. W. af Schultén, Experimentela och kliniska undersökningar beträffande Hjärnskador och deras inflytante på ögats eireulationsförhållanden. Akademisk Afhandling. Helsingfors, 1882.

13) Priestley Smith, Glancoma. London, 1879. S. 48.

14) R. Sch elske, Ueber das Verhältniss des intraocularen Druckes und der Hornhautkrümmung des Auges, v. Graefe's Arch. Bd. X. Th. 2. 1864. 